\begin{tabular}{l} 
JOURNAL Of \\
Physical Activity \\
\& Health \\
\hline
\end{tabular}

\title{
Physical activity and the risk of gallbladder disease: a systematic review and meta-analysis of cohort studies
}

\begin{tabular}{|r|l|}
\hline Journal: & Journal of Physical Activity \& Health \\
\hline Manuscript ID & JPAH.2015-0456.R1 \\
\hline Manuscript Type: & Article \\
\hline Keywords: & $\begin{array}{l}\text { epidemiology, exercise, physical activity, systematic review/meta-analysis, } \\
\text { public health, chronic disease }\end{array}$ \\
\hline \multicolumn{2}{|c}{} \\
\hline
\end{tabular}

\section{SCHOLARONE ${ }^{m}$ \\ Manuscripts}


1 Physical activity and the risk of gallbladder disease: a

2 systematic review and meta-analysis of cohort studies.

4 Short title: Physical activity and gallbladder disease

5 Manuscript type: Review

6 Keywords: Exercise, gallstones, cohort studies.

7 Word count abstract: 200

8 Word count text: 2721

9

10

11

12 
Physical activity and gallbladder disease

\section{Abstract}

2 Background: Physical activity has been hypothesized to reduce the risk of gallbladder

3 disease (gallstones, cholecystitis, cholecystectomy), however, results from epidemiological

4 studies have not always shown statistically significant associations. We conducted a

5 systematic review and meta-analysis to clarify the presence and strength of an association

6 between physical activity and gallbladder disease risk.

7

8 Methods: PubMed and Embase databases were searched for studies of physical activity and

9 gallbladder disease up to $9^{\text {th }}$ of January 2015. Prospective studies reporting relative risk (RR)

10 estimates and 95\% confidence intervals (CIs) of gallbladder disease associated with physical

11 activity were included. Summary RRs were estimated using a random effects model.

13 Results: Eight studies including 6958 cases and 218204 participants were included. The

14 summary RR for the highest versus the lowest level of physical activity was 0.75 (95\% CI:

$150.69-0.81, \mathrm{n}=8$ ) and there was no evidence of heterogeneity, $\mathrm{I}^{2}=0 \%$ ). In the dose-response

16 analysis the summary relative risk per 20 MET-hours of activity was 0.85 (95\% CI: 0.80-

$\left.170.90, \mathrm{I}^{2}=0 \%, \mathrm{n}=2\right)$ for leisure-time physical activity, $0.83\left(95 \% \mathrm{CI}: 0.76-0.90, \mathrm{I}^{2}=0 \%, \mathrm{n}=2\right)$

18 for vigorous physical activity, and $0.86\left(95 \% \mathrm{CI}: 0.76-0.98, \mathrm{I}^{2}=0 \%, \mathrm{n}=2\right)$ for non-vigorous

19 physical activity.

20

21 Conclusion: Our analysis confirms a protective effect of physical activity on risk of

22 gallbladder disease. 


\section{Introduction}

2 Gallbladder disease (including gallstones and cholecystitis) is an important cause of

3 morbidity in European and North-American populations. Of digestive diseases that require

4 hospitalization gallstones are the most frequent and costly; the economic costs of hospital

5 treatment of gallstones is over 5 billion US dollar per year. ${ }^{1}$ In the United Kingdom 49000

6 cholecystectomies are conducted every year. ${ }^{2}$ The prevalence of gallstones varies

7 substantially in sonographic surveys around the world, with prevalence rates ranging from 3-

$86 \%$ in Asian countries, 5-10\% in African countries, and 10-30\% in most European and North-

9 American populations. ${ }^{3}$ In addition, secular trend studies suggest an increase in gallstone

10 prevalence over time in Asian countries parallel to changes in lifestyle. ${ }^{4}$ These observations

11 suggest that modifiable risk factors are important in the etiology of gallstones or gallbladder

12 disease.

13 Several studies have shown that greater body fat is a strong risk factor for gallbladder

14 disease with 2-5 fold increases in risk among obese compared to lean subjects. ${ }^{5-7}$ Physical

15 activity is known to reduce the risk of metabolic abnormalities such as insulin resistance, ${ }^{8,9}$

16 elevated triglycerides, ${ }^{10}$ low levels of high-density lipoprotein cholesterol, ${ }^{10}$ diabetes, ${ }^{11}$ and

17 general and abdominal obesity, ${ }^{12-14}$ which are important risk factors for gallbladder disease. ${ }^{15-}$

$18{ }^{19}$ Several epidemiological studies have shown inverse associations between physical activity

19 and gallbladder disease, ${ }^{20-23}$ although some showed statistically non-significant

20 associations, ${ }^{19,24-26}$ but it is not known what level of physical activity is needed to

21 significantly reduce the risk. We conducted a systematic review and meta-analysis of cohort

22 studies of physical activity and risk of gallbladder disease with the aim of clarifying the

23 presence and strength of an association, the dose-response relationship and how much of the

24 association may be explained by reductions in body fatness. 
Physical activity and gallbladder disease

\section{Methods}

2

3 Search strategy

4

5 respectively) up to $9^{\text {th }}$ of January 2015 for eligible studies. As part of a larger project on risk

6 factors for gallbladder disease we used broad search terms in the PubMed search: (body mass

7 index OR BMI OR overweight OR obesity OR anthropometry OR fatness OR body fatness

8 OR abdominal fatness OR abdominal obesity OR waist circumference OR waist-to-hip ratio

9 OR physical activity OR exercise OR sports OR walking OR biking OR running OR fitness

10 OR exercise test OR inactivity OR diabetes OR smoking OR tobacco OR risk factor OR risk

11 factors) AND (gallstones OR gallbladder disease OR cholelithiasis OR cholecystectomy OR

12 cholecystitis). This search strategy was modified and adapted for the Embase search

13 (Supplementary text). We followed standard criteria for conducting and reporting meta-

14 analyses. $^{27}$ In addition, we searched the reference lists of the identified publications for

15 further studies. Study quality was assessed using the Newcastle-Ottawa scale. ${ }^{28}$

\section{Inclusion criteria and study selection}

18 We included published prospective cohort studies (which are less prone to biases than case-

19 control and cross-sectional studies) that investigated the association between physical activity

20 and the risk of gallstones, gallbladder disease or cholecystectomies. Estimates of the relative

21 risk with the $95 \%$ CIs had to be available in the publication. For the dose-response meta-

22 analysis a quantitative measure of the physical activity level had to be provided. Cross-

23 sectional and case-control studies, reviews, meta-analyses, comments, editorials, letters, news

24 articles, studies in pregnant women, and abstract and grey literature was excluded. 
1 Supplementary Table 1 shows the excluded studies and reasons for exclusions. One of the

2 included articles ${ }^{23}$ reported results from two studies, but we only included results from one of

3 these (Study of Osteoporotic Fractures) because we considered the risk estimates from the

4 other study (Walking Women Follow-up Study) to be unreliable given the low number of

5 cases and participants, with RRs of 1.00 (reference, 0 cases/42 participants), 1.08 (95\% CI:

$6 \quad 0.96-1.22,3$ cases/41 participants), and 1.13 (95\% CI: $1.01-1.28,6$ cases/41 participants) for

7 tertiles 3,2 , and 1 , respectively.

\section{Data extraction}

10 The following data were extracted from each study: The first author's last name, publication

11 year, country where the study was conducted, study period, sample size, number of

12 cases/participants, physical activity type, level of activity, relative risks and 95\% confidence

13 intervals for the highest versus the lowest level of physical activity and variables adjusted for

14 in the analysis.

\section{Statistical methods}

17 We calculated summary relative risks for the highest versus the lowest level of physical 18 activity using the random-effects model by DerSimonian and Laird, ${ }^{29}$ which takes into 19 account both within and between study variation (heterogeneity). The average of the natural 20 logarithm of the relative risks was estimated and the relative risk from each study was 21 weighted by the inverse of its variance. For one study ${ }^{26}$ which only reported a continuous 22 estimate per km running (a different measure than the other studies which were reported in 23 MET-hours per week) and which therefore could not be included in the dose-response 24 analysis, we converted the continuous estimate to an estimate that could be used for the high 25 vs. low meta-analysis by multiplying the $\ln (\mathrm{RR})$ and $\ln (\mathrm{CI})$ by 9 , and back-transforming the 
Physical activity and gallbladder disease

1 risk estimate to non-logarithmic scale. We chose to multiply by 9 because the cut-offs for

2 increasing quintiles were $<2,2-4,4-6,6-8, \geq 8 \mathrm{~km} /$ day (these were provided in a figure, but

3 categorical risk estimates were not reported), which would give midpoints in the lowest

4 category and the highest category of 1 and $9 \mathrm{~km} /$ day, respectively, assuming the open-ended

5 intervals to have the same distance as the adjacent intervals.

6 To investigate whether specific levels of physical activity were associated with

7 gallbladder disease, we used the method described by Greenland and Longnecker ${ }^{30}$ to

8 conduct dose-response analyses by computing study-specific slopes (linear trends) and 95\%

9 confidence intervals from the natural $\log$ of the relative risks and confidence intervals across

10 categories of physical activity level. The median or mean level of physical activity in each

11 category of physical activity was assigned to the corresponding relative risk for each study.

12 For studies that reported the physical activity level by ranges of activity, we estimated the

13 midpoint for each category by calculating the average of the upper and lower boundaries. We

14 conducted separate dose-response analyses for studies that reported amounts of physical

15 activity in metabolic equivalent task (MET)-hours or kcal of energy expenditure. The MET is

16 an index of the intensity of physical activity and is defined as the caloric expenditure per

17 kilogram of body weight per hour of activity divided by the equivalent per hour at rest. One

18 MET is considered equal to the energy cost of a person during quiet sitting, while walking at

19 a slow pace has a MET value of 2, and jogging and bicycling have MET values of 7-8. MET-

20 hours are the number of hours engaged in each activity multiplied with the MET value of that

21 activity, which are summed across different types of activities. Studies that did not quantify

22 the physical activity level were excluded from the dose-response analysis. We assessed a

23 potential nonlinear dose-response relationship between physical activity and gallbladder

24 disease using fractional polynomial models. ${ }^{31}$ We determined the best fitting second order

25 fractional polynomial regression model, defined as the one with the lowest deviance. A 
2

1 likelihood ratio test was used to assess the difference between the nonlinear and linear models

2 to test for nonlinearity. ${ }^{32}$

3 Heterogeneity between studies was evaluated using $\mathrm{Q}$ and $\mathrm{I}^{2}$ statistics. ${ }^{33} \mathrm{I}^{2}$ is the 4 proportion of total variation contributed by between-study variation rather than chance. $\mathrm{I}^{2}$ -

5 values of $25 \%, 50 \%$ and $75 \%$ indicate low, moderate and high heterogeneity, respectively.

6 We conducted main analyses and subgroup analyses by study characteristics such as gender,

7 type of outcome (gallstones, gallbladder disease and cholecystectomy), number of cases,

8 geographic location and adjustment for confounding factors. Study quality was assessed

9 using the Newcastle-Ottawa scale which rates the studies from 0 to 9 by assessing the 10 selection, comparability and outcome of each study. ${ }^{28}$

by inspection of funnel plots. The statistical analyses were conducted using the software package Stata, version 10.1 software (StataCorp, Texas, US). 
Physical activity and gallbladder disease

\section{Results}

2

3 We identified 8 cohort studies that could be included in the analysis of physical activity and

4 gallbladder disease ${ }^{19-26}$, with a total of 6958 cases and 218204 participants (Figure 1, Table

5 1). Seven of the studies were from North-America and one was from Europe.

6

$7 \quad$ High versus low analysis

8 Eight cohort studies ${ }^{19-26}$ investigated the association between physical activity and

9 gallbladder disease risk (Table 1). The summary relative risk for high versus low physical

10 activity was 0.75 (95\% CI: $0.69-0.81)$ with no evidence of heterogeneity, $\mathrm{I}^{2}=0 \%$,

11 pheterogeneity $=0.94$ (Figure 2). There was some evidence of publication bias with Egger's test,

$12 \mathrm{p}=0.02$, but not with Begg's test, $\mathrm{p}=0.90$ (Supplementary Figure 1), but this disappeared

13 when we restricted the analysis to studies with $\geq 500$ cases, $p=0.14$, and the results were

14 similar, showing a summary RR of 0.76 (95\% CI: $0.69-0.84, \mathrm{I}^{2}=0 \%$, pheterogeneity $=0.62$ ).

Dose-response analyses

17 Only two cohort studies could be included in the dose-response analysis of leisure-time

18 physical activity in MET-hours per week. ${ }^{21,22}$ The summary relative risk was 0.85 (95\% CI:

$190.80-0.90, \mathrm{I}^{2}=0 \%, p_{\text {heterogeneity }}=0.84$ ) per 20 MET-hours per week (Supplementary Figure 2a).

20 When BMI was omitted from the multivariate adjustment the summary RR was 0.78 (95\%

21 CI: $0.73-0.83, I^{2}=0 \%$, pheterogeneity $=0.54$ ) (Figure $\left.2 a\right)$. There was no evidence of a nonlinear

22 association between leisure-time physical activity and gallbladder disease, $p_{\text {nonlinearity }}=0.28$

23 (Figure 2b, Supplementary Table 2). Two cohort studies ${ }^{23,24}$ could be included in the dose-

24 response analysis of energy expended on physical activity per week (kcal per week). The 
1 summary relative risk per $1000 \mathrm{kcal} /$ week was 0.93 (95\% CI: $0.74-1.16, \mathrm{I}^{2}=88.7 \%$,

$\left.2 \quad p_{\text {heterogeneity }}=0.003\right)($ Supplementary Figure 3).

3

$4 \quad$ Vigorous and non-vigorous physical activity

5 Only two cohort studies could be included in the dose-response analysis of vigorous (defined

6 as activity that required 6 or more METs per hour) and non-vigorous (defined as activity that

7 required less than 6 METs per hour, including light and moderate activity) physical

8 activity. $^{21,22}$ The summary RR per 20 MET-hours per week was 0.83 (95\% CI: 0.76-0.90,

$9 \mathrm{I}^{2}=0 \%$, pheterogeneity $\left.=0.85\right)$ for vigorous physical activity and it was 0.86 (95\% CI: $0.76-0.98$,

$10 \mathrm{I}^{2}=0 \%, p_{\text {heterogeneity }}=0.45$ ) for non-vigorous physical activity (Supplementary Figure $4 \mathrm{a}$,

11 Supplementary Figure 5a). When BMI was omitted from the multivariate adjustment the

12 respective summary RRs were $0.76\left(95 \% \mathrm{CI}: 0.70-0.83, \mathrm{I}^{2}=0 \%\right.$, pheterogeneity $\left.=0.94\right)$ and 0.78

$13\left(95 \%\right.$ CI: $\left.0.65-0.93, I^{2}=36 \%, p_{\text {heterogeneity }}=0.21\right)$ (Supplementary Figure 4a, Supplementary

14 Figure 5a). There was evidence of a nonlinear association between vigorous and non-

15 vigorous physical activity and gallbladder disease, $p_{\text {nonlinearity }}=0.009$ and $p_{\text {nonlinearity }}=<0.0001$,

16 respectively, with a steeper inverse association at higher levels of vigorous physical activity,

17 while the association for non-vigorous physical activity was less pronounced at higher levels

18 of activity (Supplementary Figure 4b, Supplementary Figure 5b, Supplementary Table 2).

\section{Subgroup and sensitivity analyses}

21 Relations of physical activity to gallbladder disease were inverse in all strata of subgroup 22 analyses, defined by gender, duration of follow-up, geographic location, type of outcome, 23 number of cases, study quality and adjustment for confounding factors (Table 2). With meta24 regression analyses there was no evidence that the results differed between these subgroups 25 (Table 2). Mean and median study quality scores were 6.13 and 6.5, respectively. When we 
Physical activity and gallbladder disease

32

33

34

35

36

1 conducted sensitivity analyses removing one study at a time, the size of the summary RR

2 ranged from 0.71 (95\% CI: $0.63-0.80)$ when excluding the study by Leitzmann et $\mathrm{al}^{22}$ to 0.76

3 (95\% CI: 0.70-0.82) when excluding the study by Storti et al. ${ }^{23}$

\section{4}

5

6

7 
Discussion

To our knowledge this is the first meta-analysis of observational studies of physical

3 activity and risk of gallbladder disease. Our results confirm an inverse association between

4 higher levels of physical activity and gallbladder disease. There was a $25 \%$ relative risk

5 reduction for participants with the highest versus the lowest physical activity level. Both

$6 \quad$ vigorous and non-vigorous physical activity was associated with reduced risk.

The present systematic review and meta-analysis has some limitations that need to be

8 discussed. The number of studies included was modest and few studies could be included in

9 the dose-response analysis because the physical activity level was not quantified or it was

10 quantified in a manner that was not possible to combine with the remaining studies. Further

11 studies should aim to report results in a manner that can be included in future updated dose-

12 response meta-analyses (for example in MET-hrs per week). Our analysis suggests that both

13 vigorous and non-vigorous physical activity reduces risk of gallbladder disease, although the

14 dose-response relationship appeared to be stronger for vigorous physical activity than for

15 non-vigorous physical activity. This might at least partly be due to the greater range of

16 vigorous physical activity than of non-vigorous activity. However, further studies are needed

17 as the number of studies on subtypes of physical activity was low. Many of the included

18 studies adjusted for important confounding factors and in subgroup analyses, we did not find

19 evidence that the results differed when stratified by adjustment for confounding factors. We

20 cannot, however, exclude the possibility that residual confounding by other unidentified risk

21 factors could have partly affected the results. Physical activity was assessed using self-

22 reported questionnaires and therefore measurement error in the exposure variable is likely to

23 have occurred. Two studies made corrections for measurement error using data from their

24 validation studies. ${ }^{21,22}$ One of the studies reported a RR of 0.81 (95\% CI: $\left.0.73-0.89\right)$ per 25

25 MET-hours per week of moderate intensity exercise in an uncorrected model, and 0.64 (95\% 
1 CI: 0.49-0.86) in a model corrected for measurement error, ${ }^{21}$ and another study reported a RR

2 of 0.85 (95\% CI: 0.80-0.90) per 25 MET-hours per week in an uncorrected model and 0.58

3 (95\% CI: 0.43-0.77) in a model corrected for measurement error, ${ }^{22}$ suggesting substantial

4 underestimation of the RR due to measurement errors. In addition, because of the limited

5 number of studies we chose to analyse leisure-time physical activity together with total

6 physical activity. However, the magnitude of the risk estimates were similar in stratified

7 analyses, and there was no heterogeneity between these subgroups. There were some

8 differences in the definition of the outcome as well. Some studies investigated only

9 gallstones, while others investigated gallbladder disease which included both gallstones and

10 inflammation of the gallbladder (cholecystitis), and in one study the outcome was surgical

11 removal of the gallbladder (cholecystectomy), while no cohort studies reported on

12 cholecystitis only. However, risk estimates where similar when stratified by type of outcome

13 and there was no evidence of heterogeneity between these subgroups. Because most of the

14 studies were from North America and included mainly Caucasian subjects, further studies

15 among other ethnicities and in other geographic locations are needed to clarify if the findings

16 also apply to other populations. One last limitation is that the study selection was done by one

17 author and not in duplicate.

18 Several biological mechanisms could explain a protective effect of physical activity

19 on risk of gallbladder disease. Physical activity has been established to protect against general

20 and abdominal overweight and obesity ${ }^{12-14}$ and diabetes ${ }^{36}$ which are strongly associated with

21 risk of gallbladder disease..$^{5,7,16,17}$ However, in this meta-analysis most studies adjusted for

22 BMI and several studies also adjusted for diabetes and the results persisted in these subgroup

23 analyses. In two studies ${ }^{21,22}$ that provided risk estimates both adjusted and not adjusted for

24 BMI the inverse association was slightly more pronounced when BMI was omitted from the

25 multivariable models compared to when adjusted for BMI (RR of 0.78 vs. 0.85 per 25 MET- 
1 hours/week for leisure-time activity), suggesting that reduced adiposity may explain

2 approximately $30-35 \%$ of the reduction in risk observed with physical activity. However, a

3 clinically relevant reduction in risk was observed even with adjustment for BMI and other

4 mechanisms are therefore likely to be involved in explaining the beneficial effects of physical

5 activity on gallbladder disease. Physical activity has been shown to reduce levels of total

6 cholesterol, ${ }^{10}$ triglycerides, ${ }^{10}$ and insulin resistance ${ }^{8,9}$ and this may further contribute to

7 reduced gallbladder disease risk. In addition, physical activity may influence gallbladder

8 disease risk by modulating gallbladder motility. ${ }^{37}$ Hypomotility of the gallbladder leads to

9 incomplete emptying of the gallbladder which promotes bile stasis and crystal formation

10 leading to gallstones. ${ }^{38}$ Physical activity may also increase vagal tone which can stimulate

11 gallbladder emptying. ${ }^{39}$

12 Strengths of this analysis include the large sample size which provides sufficient

13 statistical power to detect modest associations, and comprehensive subgroup, sensitivity and

14 dose-response analyses, as well as moderate to high study quality. Because this analysis only

15 included cohort studies recall bias is not likely to explain the findings and there is less

16 possibility for selection bias. When stratified by gender, similar reductions in risk were

17 observed for men and women.

18 In conclusion, the results from this systematic review and meta-analysis confirm that

19 a high level of physical activity reduces the risk of gallbladder disease. This adds to the many

20 health benefits that have already been documented for physical activity. Future studies should

21 attempt to further define the dose-response relationship between physical activity and specific

22 types of physical activity and gallbladder disease risk and additional studies in non-American

23 populations are warranted. 
1 Acknowledgement/Contribution: DA designed the research, conducted the literature search

2 and analyses and wrote the first draft of the paper. All authors interpreted the data, revised the

3 subsequent drafts for important intellectual content, read and approved the final manuscript.

4

5 Conflict of interest: The authors declare that there is no duality of interest associated with

6 this manuscript.

7

8 Funding source: This work was funded by "Liaison Committee between the Central Norway

9 Regional Health Authority (RHA) and the Norwegian University of Science and Technology

10 (NTNU)".

11

12

13

14

15

16

17

18

19

20 
(1) Sandler RS, Everhart JE, Donowitz M et al. The burden of selected digestive diseases in the United States. Gastroenterology. 2002;122:1500-1511. [PubMed: 11984534]

(2) Sanders G, Kingsnorth AN. Gallstones. BMJ. 2007;335:295-299. [PubMed: 17690370]

(4) Park YH, Park SJ, Jang JY et al. Changing patterns of gallstone disease in Korea. World J

(5) Stampfer MJ, Maclure KM, Colditz GA, Manson JE, Willett WC. Risk of symptomatic gallstones in women with severe obesity. Am J Clin Nutr. 1992;55:652-658. [PubMed: 1550039]

(6) Liu B, Balkwill A, Spencer E, Beral V. Relationship between body mass index and length of hospital stay for gallbladder disease. J Public Health (Oxf). 2008;30:161-166. [PubMed: $18308742]$

(7) Aune D, Norat T, Vatten LJ. Body mass index, abdominal fatness and the risk of gallbladder disease. Eur J Epidemiol. 2015;30:1009-1019. [PubMed: 26374741]

(8) Riserus U, Arnlov J, Berglund L. Long-term predictors of insulin resistance: role of lifestyle and metabolic factors in middle-aged men. Diabetes Care. 2007;30:2928-2933. [PubMed: 17644620] 
Physical activity and gallbladder disease

1

2

3

4

5

6

7

8

9

10

11

12

13

14

15

16

17

18

19

20

(9) Assah FK, Brage S, Ekelund U, Wareham NJ. The association of intensity and overall level of physical activity energy expenditure with a marker of insulin resistance. Diabetologia. 2008;51:1399-1407. [PubMed: 18488189]

(10) Kelley GA, Kelley KS, Tran ZV. Aerobic exercise and lipids and lipoproteins in women: a meta-analysis of randomized controlled trials. J Womens Health (Larchmt). 2004;13:11481164. [PubMed: 15650348]

(11) Jeon CY, Lokken RP, Hu FB, van Dam RM. Physical activity of moderate intensity and risk of type 2 diabetes: a systematic review. Diabetes Care. 2007;30:744-752. [PubMed: $17327354]$

(12) Mozaffarian D, Hao T, Rimm EB, Willett WC, Hu FB. Changes in diet and lifestyle and longterm weight gain in women and men. $N$ Engl J Med. 2011;364:2392-2404. [PubMed: 21696306]

(13) Ekelund U, Besson H, Luan J et al. Physical activity and gain in abdominal adiposity and body weight: prospective cohort study in 288,498 men and women. Am J Clin Nutr. 2011;93:826-835. [PubMed: 21346093]

(14) Davidson LE, Tucker L, Peterson T. Physical activity changes predict abdominal fat change in midlife women. J Phys Act Health. 2010;7:316-322. [PubMed: 20551487]

(15) Liu CM, Tung TH, Tsai ST et al. Serum insulin, insulin resistance, beta-cell dysfunction, and gallstone disease among type 2 diabetics in Chinese population: a community-based study in Kinmen, Taiwan. World J Gastroenterol. 2005;11:7159-7164. [PubMed: 16437664] 
1

2

3

4

5

6

7

8

9

10

11

12

13

14

15

16

17

18

19

20

(16) Jamal MM, Yoon EJ, Vega KJ, Hashemzadeh M, Chang KJ. Diabetes mellitus as a risk factor for gastrointestinal cancer among American veterans. World J Gastroenterol. 2009;15:52745278. [PubMed: 19908334]

(17) Aune D, Vatten LJ. Diabetes mellitus and the risk of gallbladder disease: A systematic review and meta-analysis of prospective studies. J Diabetes Complications. 2015. doi: 10.1016/j.jdiacomp.2015.11.012. [Epub ahead of print] [PubMed: 26684168]

(18) Tsai CJ, Leitzmann MF, Willett WC, Giovannucci EL. Prospective study of abdominal adiposity and gallstone disease in US men. Am J Clin Nutr. 2004;80:38-44. [PubMed: $15213025]$

(19) Banim PJ, Luben RN, Wareham NJ, Sharp SJ, Khaw KT, Hart AR. Physical activity reduces the risk of symptomatic gallstones: a prospective cohort study. Eur J Gastroenterol Hepatol. 2010;22:983-988. [PubMed: 20130468]

(20) Kato I, Nomura A, Stemmermann GN, Chyou PH. Prospective study of clinical gallbladder disease and its association with obesity, physical activity, and other factors. Dig Dis Sci. 1992;37:784-790. [PubMed: 1563324]

(21) Leitzmann MF, Giovannucci EL, Rimm EB et al. The relation of physical activity to risk for symptomatic gallstone disease in men. Ann Intern Med. 1998;128:417-425. [PubMed: 1563324]

(22) Leitzmann MF, Rimm EB, Willett WC et al. Recreational physical activity and the risk of cholecystectomy in women. N Engl J Med. 1999;341:777-784. [PubMed 10477775] 
Physical activity and gallbladder disease

1

2

3

4

5

6

7

8

9

10

11

12

13

14

15

16

17

18

19

20

(23) Storti KL, Brach JS, FitzGerald SJ, Zmuda JM, Cauley JA, Kriska AM. Physical activity and decreased risk of clinical gallstone disease among post-menopausal women. Prev Med. 2005;41:772-777. [PubMed: 16038966]

(24) Sahi T, Paffenbarger RS, Jr., Hsieh CC, Lee IM. Body mass index, cigarette smoking, and other characteristics as predictors of self-reported, physician-diagnosed gallbladder disease in male college alumni. Am J Epidemiol. 1998;147:644-651. [PubMed: 9554603]

(25) Boland LL, Folsom AR, Rosamond WD. Hyperinsulinemia, dyslipidemia, and obesity as risk factors for hospitalized gallbladder disease. A prospective study. Ann Epidemiol. 2002;12:131-140. [PubMed: 11880221]

(26) Williams PT. Independent effects of cardiorespiratory fitness, vigorous physical activity, and body mass index on clinical gallbladder disease risk. Am J Gastroenterol. 2008;103:22392247. [PubMed 18637096]

(27) Stroup DF, Berlin JA, Morton SC et al. Meta-analysis of observational studies in epidemiology: a proposal for reporting. Meta-analysis Of Observational Studies in Epidemiology (MOOSE) group. JAMA. 2000;283:2008-2012. [PubMed: 10789670]

(28) Wells G, Shea B, O'Connell D. et al. The Newcastle-Ottawa Scale (NOS) for assessing the quality of nonrandomised studies in meta-analyses. $\underline{h t p: / / w w w}$ ohri.ca/programs/clinical_epidemiology/oxford.asp, Accessed 13.08.2014.

(29) DerSimonian R, Laird N. Meta-analysis in clinical trials. Control Clin Trials. 1986;7:177188. [PubMed: 3802833] 
1

2

3

4

5

6

7

8

9

10

(30) Greenland S, Longnecker MP. Methods for trend estimation from summarized dose-response data, with applications to meta-analysis. Am J Epidemiol. 1992;135:1301-1309. [PubMed: 1626547]

(31) Royston P. A strategy for modelling the effect of a continuous covariate in medicine and epidemiology. Stat Med. 2000;19:1831-1847. [PubMed: 10867674]

(32) Bagnardi V, Zambon A, Quatto P, Corrao G. Flexible meta-regression functions for modeling aggregate dose-response data, with an application to alcohol and mortality. Am J Epidemiol. 2004;159:1077-1086. [PubMed: 15155292]

(33) Higgins JP, Thompson SG. Quantifying heterogeneity in a meta-analysis. Stat Med. 2002;21:1539-1558. [PubMed: 12111919]

(34) Egger M, Davey SG, Schneider M, Minder C. Bias in meta-analysis detected by a simple, graphical test. BMJ. 1997;315:629-634. [PubMed: 9310563]

(35) Begg CB, Mazumdar M. Operating characteristics of a rank correlation test for publication bias. Biometrics. 1994;50:1088-1101. [PubMed: 7786990]

(36) Aune D, Norat T, Leitzmann M, Tonstad S, Vatten LJ. Physical activity and the risk of type 2 diabetes: a systematic review and dose-response meta-analysis. Eur J Epidemiol. 2015;30:529-542. [PubMed: 26092138]

(37) Sari R, Balci N, Balci MK. Effects of exercise on gallbladder volume and motility in obese women. J Clin Ultrasound. 2005;33:218-222. [PubMed: 16047386] 
Physical activity and gallbladder disease

1

2

3

4

5

6

7

8

9

10

11

12

(38) Bowen JC, Brenner HI, Ferrante WA, Maule WF. Gallstone disease. Pathophysiology, epidemiology, natural history, and treatment options. Med Clin North Am. 1992;76:1143-

1157. [PubMed: 1518331]

(39) Krotkiewski M, Bjorntorp P, Holm G et al. Effects of physical training on insulin, connecting peptide (C-peptide), gastric inhibitory polypeptide (GIP) and pancreatic polypeptide (PP)

levels in obese subjects. Int J Obes. 1984;8:193-199. [PubMed: 6378815] 
Table 1. Prospective studies of physical activity and gallbladder disease

\begin{tabular}{|c|c|c|c|c|c|c|}
\hline $\begin{array}{l}\text { Number of } \\
\text { participants, } \\
\text { number of } \\
\text { cases }\end{array}$ & $\begin{array}{l}\text { Study } \\
\text { period, } \\
\text { duration of } \\
\text { follow-up }\end{array}$ & $\begin{array}{l}\text { Study } \\
\text { quality } \\
\text { score }\end{array}$ & Physical activity exposure & Quantity of physical activity & $\begin{array}{l}\text { Relative risk } \\
(95 \% \\
\text { confidence } \\
\text { interval })\end{array}$ & Adjustment for confounders \\
\hline $\begin{array}{l}7831 \text { Japanese } \\
\text { men, age } \geq 45 \text { - } \\
65: 471 \\
\text { gallbladder } \\
\text { disease }\end{array}$ & $\begin{array}{l}1965-68- \\
1990\end{array}$ & 8 & Physical activity index (TPA) & $\geq 35.5$ vs. $<29.5 \mathrm{METs} / \mathrm{d}$ & $0.7(0.6-1.0)$ & $\begin{array}{l}\text { Age, pack-years of cigarettes, } \\
\text { alcohol, serum TG, serum uric } \\
\text { acid, serum glucose, DBP, height, } \\
\text { BMI, total calories }\end{array}$ \\
\hline $\begin{array}{l}16414 \text { men, } \\
\text { age } 32-74 \\
\text { years: } 268 \\
\text { gallbladder } \\
\text { disease cases }\end{array}$ & $\begin{array}{l}\text { 1962-1966- } \\
1977, \text { up to } \\
15 \text { years } \\
\text { follow-up }\end{array}$ & 7 & Physical activity index (LTPA) & $\geq 3500$ vs. $<500 \mathrm{kcal} / \mathrm{wk}$ & $0.72(0.46-1.13)$ & $\begin{array}{l}\text { Age, calendar year, BMI, BMI } \\
\text { change between college and } \\
\text { 1962/66, smoking habit }\end{array}$ \\
\hline $\begin{array}{l}45813 \text { men, } \\
\text { age } 40-75 \\
\text { years: } 828 \\
\text { symptomatic } \\
\text { gallstone } \\
\text { cases }\end{array}$ & $\begin{array}{l}\text { 1986-1994, } \\
8 \text { years } \\
\text { follow-up }\end{array}$ & 7 & $\begin{array}{l}\text { Total physical activity (LTPA) } \\
\text { Total physical activity (LTPA) } \\
\text { Total physical activity (LTPA), BMI adj. } \\
\text { Vigorous activity } \\
\text { Vigorous activity } \\
\text { Vigorous activity, BMI adj. } \\
\text { Nonvigorous activity } \\
\text { Nonvigorous activity } \\
\text { Nonvigorous activity, BMI adj. }\end{array}$ & $\begin{array}{l}\geq 32.6 \text { vs. } 0-2.7 \text { MET-hrs/wk } \\
\text { Per } 25 \text { MET-hrs/wk } \\
\geq 32.6 \text { vs. } 0-2.7 \text { MET-hrs/wk } \\
\geq 25.1 \text { vs. } 0 \text { MET-hrs/wk } \\
\text { Per } 25 \text { MET-hrs/wk } \\
\geq 25.1 \text { vs. } 0 \text { MET-hrs/wk } \\
\geq 11.4 \text { vs. } 0-0.8 \mathrm{MET}-\mathrm{hrs} / \mathrm{wk} \\
\text { Per } 25 \mathrm{MET}-\mathrm{hrs} / \mathrm{wk} \\
\geq 11.4 \text { vs. } 0-0.8 \mathrm{MET}-\mathrm{hrs} / \mathrm{wk}\end{array}$ & $\begin{array}{l}0.63(0.51-0.79) \\
0.81(0.73-0.89) \\
0.72(0.59-0.90) \\
0.58(0.45-0.75) \\
0.77(0.67-0.88) \\
0.65(0.50-0.85) \\
0.93(0.75-1.15) \\
0.87(0.73-1.03) \\
0.97(0.78-1.19) \\
\end{array}$ & $\begin{array}{l}\text { Age, diabetes mellitus, smoking, } \\
\text { cholesterol-lowering drugs, } \\
\text { thiazide use, NSAIDS, alcohol, } \\
\text { dietary fiber, carbohydrates }\end{array}$ \\
\hline $\begin{array}{l}60290 \\
\text { women, age } \\
40-65 \text { years: } \\
3257 \\
\text { cholecystec- } \\
\text { tomies }\end{array}$ & $\begin{array}{l}1986-1996, \\
10 \text { years } \\
\text { follow-up }\end{array}$ & 7 & $\begin{array}{l}\text { Physical activity (LTPA) } \\
\text { Physical activity, BMI/weight change-adj. } \\
\text { Physical activity, BMI/weight change-adj. } \\
\text { Vigorous physical activity } \\
\text { Vigorous physical activity, BMI/weight } \\
\text { change-adj. } \\
\text { Vigorous physical activity, BMI/weight } \\
\text { change-adj. } \\
\text { Nonvigorous physical activity } \\
\text { Nonvigorous physical activity, BMI/weight }\end{array}$ & $\begin{array}{l}\geq 22.1 \text { vs. } 0-1.6 \mathrm{MET}-\mathrm{hrs} / \mathrm{wk} \\
\geq 22.1 \text { vs. } 0-1.6 \mathrm{MET}-\mathrm{hrs} / \mathrm{wk} \\
\text { Per } 25 \mathrm{MET}-\mathrm{hrs} / \mathrm{wk} \\
\geq 16 \text { vs. } 0 \mathrm{MET}-\mathrm{hrs} / \mathrm{wk} \\
\geq 16 \text { vs. } 0 \mathrm{MET}-\mathrm{hrs} / \mathrm{wk} \\
\text { Per } 10 \mathrm{MET}-\mathrm{hrs} / \mathrm{wk} \\
\geq 10.8 \text { vs. } 0-0.8 \mathrm{MET}-\mathrm{hrs} / \mathrm{wk} \\
\geq 10.8 \text { vs. } 0-0.8 \mathrm{MET}-\mathrm{hrs} / \mathrm{wk}\end{array}$ & $\begin{array}{l}0.69(0.61-0.78) \\
0.79(0.71-0.89) \\
0.85(0.80-0.90) \\
0.75(0.66-0.85) \\
0.82(0.73-0.93) \\
0.89(0.86-0.94) \\
0.86(0.77-0.98) \\
0.95(0.84-1.06)\end{array}$ & $\begin{array}{l}\text { Age, parity, OC use, use of HRT, } \\
\text { diabetes mellitus, pack-years of } \\
\text { smoking, use of cholesterol- } \\
\text { lowering drugs, thiazide use, } \\
\text { NSAIDS, dietary fiber, } \\
\text { carbohydrates, alcohol, coffee }\end{array}$ \\
\hline
\end{tabular}


Physical activity and gallbladder disease

\begin{tabular}{|c|c|c|c|c|c|c|c|}
\hline $\begin{array}{l}5 \\
6 \\
7\end{array}$ & & & & $\begin{array}{l}\text { change-adj. } \\
\text { Nonvigorous physical activity, BMI/weight } \\
\text { change-adj. }\end{array}$ & Per 10 MET-hrs/wk & $0.91(0.87-0.96)$ & \\
\hline $\begin{array}{l}8 \text { Boland LL } \\
9 \text { et al, } \\
102002, \\
11 \text { USA } \\
12 \\
13\end{array}$ & $\begin{array}{l}12773 \text { men } \\
\text { and women, } \\
\text { age } 45-64 \\
\text { years: } 397 \\
\text { gallbladder } \\
\text { disease }\end{array}$ & $\begin{array}{l}1987-1989- \\
1996,8.2 \\
\text { years } \\
\text { follow-up }\end{array}$ & 6 & \begin{tabular}{|l|} 
Sport index (LTPA), men \\
Sport index (LTPA), women
\end{tabular} & $\begin{array}{l}\geq 3.25 \text { vs. } \leq 1.75 \\
\geq 3.25 \text { vs. } \leq 1.75\end{array}$ & $\begin{array}{l}0.81(0.5-1.3) \\
0.72(0.5-1.1)\end{array}$ & Age \\
\hline $\begin{array}{l}1 \text { \$torti KL } \\
1 \text { ftal, } 2005 \\
16^{\text {USA }} \\
17 \\
\end{array}$ & $\begin{array}{l}8010 \text { women, } \\
\text { age } \geq 65 \text { years: } \\
1281 \text { gallstone } \\
\text { disease cases }\end{array}$ & $\begin{array}{l}\text { 1989-90- } \\
1992-1994, \\
\sim 3.5 \text { years } \\
\text { follow-up } \\
\end{array}$ & 5 & $\begin{array}{l}\text { Physical activity (LTPA) } \\
\text { Physical activity (LTPA) } \\
\end{array}$ & $\begin{array}{l}\text { Per } 500 \mathrm{kcal} / \mathrm{wk} \\
>2244 \text { vs. }<512 \mathrm{kcal} / \mathrm{wk}\end{array}$ & $\begin{array}{l}0.94(0.90-0.98) \\
0.63(0.44-0.90)\end{array}$ & $\begin{array}{l}\text { Age, BMI, education, hormone use, } \\
\text { diabetes prevalence, smoking } \\
\text { status, alcohol use, parity }\end{array}$ \\
\hline $\begin{array}{l}18 \text { Villiams } \\
19 \text { PT et al, } \\
20 \text { 2008, } \\
21 \\
22 \\
23 \\
23 \\
24\end{array}$ & $\begin{array}{l}29110 \text { male } \\
\text { and } 11953 \\
\text { female } \\
\text { runners, mean } \\
\text { age } 45 / 39 \\
\text { years: } 166 \\
\text { gallbladder } \\
\text { disease cases }\end{array}$ & $\begin{array}{l}\text { 1991-94- } \\
2002,7.6 \\
\text { years } \\
\text { follow-up }\end{array}$ & 4 & $\begin{array}{l}\text { Physical activity (LTPA), men } \\
\text { Physical activity (LTPA), men, BMI adj. } \\
\text { Physical activity (LTPA), women } \\
\text { Physical activity (LTPA), women, BMI adj. }\end{array}$ & $\begin{array}{l}\text { Per km/d } \\
\text { Per km/d } \\
\text { Per km/d } \\
\text { Per km/d }\end{array}$ & $\begin{array}{l}0.93(0.87-0.98) \\
0.97(0.91-1.02) \\
0.91(0.84-0.97) \\
0.96(0.89-1.03)\end{array}$ & $\begin{array}{l}\text { Age, race, education, pack-yrs of } \\
\text { cigarette use, weekly intakes of } \\
\text { meat, fish, fruit, alcohol, parity }\end{array}$ \\
\hline $\begin{array}{l}25 \text { Banim PJR } \\
\text { 26t al, 2010, } \\
2 \text { Jnited } \\
26 \text { Kingdom } \\
29 \\
30\end{array}$ & $\begin{array}{l}25639 \text { men } \\
\text { and women, } \\
\text { age } 40-74 \\
\text { years: } 290 \\
\text { gallstone } \\
\text { cases }\end{array}$ & $\begin{array}{l}1993-1997 \\
-2007,14 \\
\text { years } \\
\text { follow-up }\end{array}$ & 8 & \begin{tabular}{|l} 
Physical activity (TPA), all \\
Physical activity (TPA), men \\
Physical activity (TPA), women
\end{tabular} & $\begin{array}{l}\text { Active vs. inactive } \\
\text { Active vs. inactive } \\
\text { Active vs. inactive }\end{array}$ & $\begin{array}{l}0.75(0.50-1.12) \\
0.81(0.43-1.51) \\
0.66(0.38-1.13)\end{array}$ & $\begin{array}{l}\text { Age, alcohol, BMI, sex } \\
\text { Women: also adjusted for parity } \\
\text { and HRT }\end{array}$ \\
\hline
\end{tabular}

$\mathrm{BMI}=$ Body mass index, $\mathrm{DM}=$ diabetes mellitus, $\mathrm{HRT}=$ hormone replacement therapy, $\mathrm{LTPA}=$ leisure-time physical activity, MUFA=monounsaturated fatty acids, $\mathrm{NA}=$ not available, NSAID=non-steroidal anti-inflammatory drugs, $\mathrm{OC}$ use $=$ oral contraceptive use, $\mathrm{SFA}=$ saturated fatty acids, $\mathrm{TPA}=$ total physical activity (leisure + occupational activity) 
Table 2. Subgroup analyses of physical activity and gallbladder disease

\begin{tabular}{|c|c|c|c|c|c|}
\hline & \multicolumn{5}{|c|}{ Physical activity and gallbladder disease } \\
\hline & $n$ & Relative risk $(95 \% \mathrm{CI})$ & $I^{2}(\%)$ & $P_{\mathrm{h}}{ }^{1}$ & $P_{\mathrm{h}}^{2}$ \\
\hline All studies & 8 & $0.75(0.69-0.81)$ & 0 & 0.94 & \\
\hline \multicolumn{6}{|l|}{ Gender } \\
\hline Men & 6 & $0.73(0.63-0.84)$ & 0 & 0.99 & 0.60 \\
\hline Women & 5 & $0.76(0.69-0.85)$ & 0 & 0.75 & \\
\hline \multicolumn{6}{|l|}{ Outcome } \\
\hline Gallstones & 3 & $0.70(0.59-0.82)$ & 0 & 0.82 & 0.24 \\
\hline Gallbladder disease & 4 & $0.73(0.62-0.85)$ & 0 & 0.98 & \\
\hline Cholecystectomy & 1 & $0.79(0.71-0.88)$ & & & \\
\hline \multicolumn{6}{|l|}{ Type of physical activity } \\
\hline Leisure-time physical activity & 6 & $0.76(0.70-0.83)$ & 0 & 0.87 & 0.51 \\
\hline Total physical activity & 2 & $0.70(0.57-0.86)$ & 0 & 1.00 & \\
\hline \multicolumn{6}{|l|}{ Geographic location } \\
\hline Europe & 1 & $0.70(0.48-1.02)$ & & & 0.72 \\
\hline America & 7 & $0.75(0.69-0.82)$ & 0 & 0.90 & \\
\hline \multicolumn{6}{|l|}{ Number of cases } \\
\hline Cases $<500$ & 4 & $0.72(0.62-0.85)$ & 0 & 0.98 & 0.43 \\
\hline Cases 500-<1000 & 2 & $0.72(0.59-0.88)$ & 28.4 & 0.24 & \\
\hline Cases $\geq 1000$ & 2 & $0.72(0.59-0.88)$ & 0 & 1.00 & \\
\hline \multicolumn{6}{|l|}{ Study quality } \\
\hline $0-3$ stars & 0 & & & & 0.16 \\
\hline 4-6 stars & 4 & $0.71(0.59-0.85)$ & 0 & 0.88 & \\
\hline $7-9$ stars & 4 & $0.76(0.69-0.83)$ & 0 & 0.74 & \\
\hline \multicolumn{6}{|l|}{ Adjustment for confounding factors ${ }^{3}$} \\
\hline \multirow[t]{2}{*}{ Age } & 8 & $0.75(0.69-0.81)$ & 0 & 0.94 & $\mathrm{NC}$ \\
\hline & 0 & & & & \\
\hline
\end{tabular}




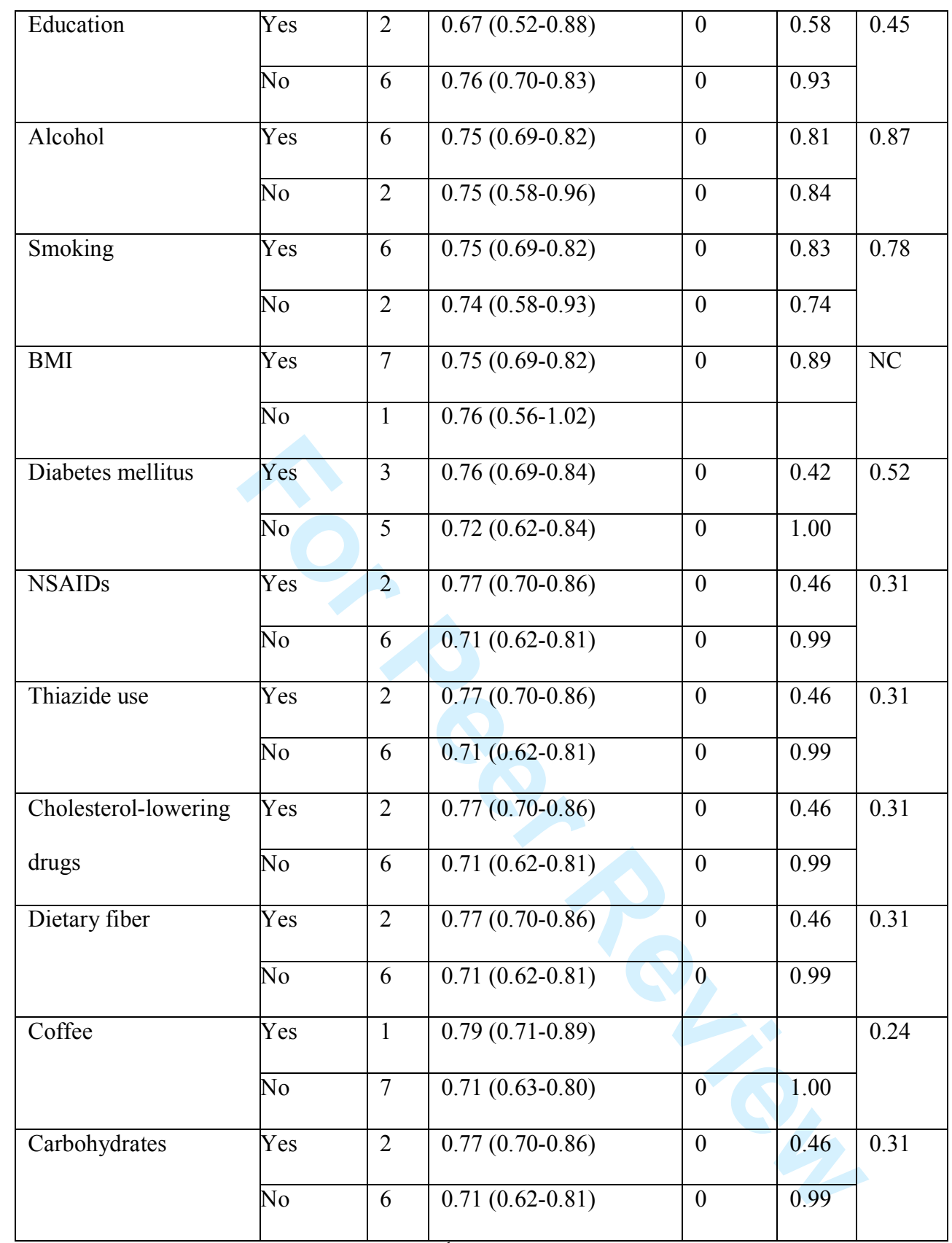

$n$ denotes the number of studies, ${ }^{1} \mathrm{P}$ for heterogeneity within each subgroup,

${ }^{2} \mathrm{P}$ for heterogeneity between subgroups with meta-regression analysis

$\mathrm{NC}$, not calculable because no studies were present in one of the subgroups. 
Figure 1. Flow-chart of study selection

\section{7 records identified in total: 7799 records identified in PubMed 4948 records identified in Embase}

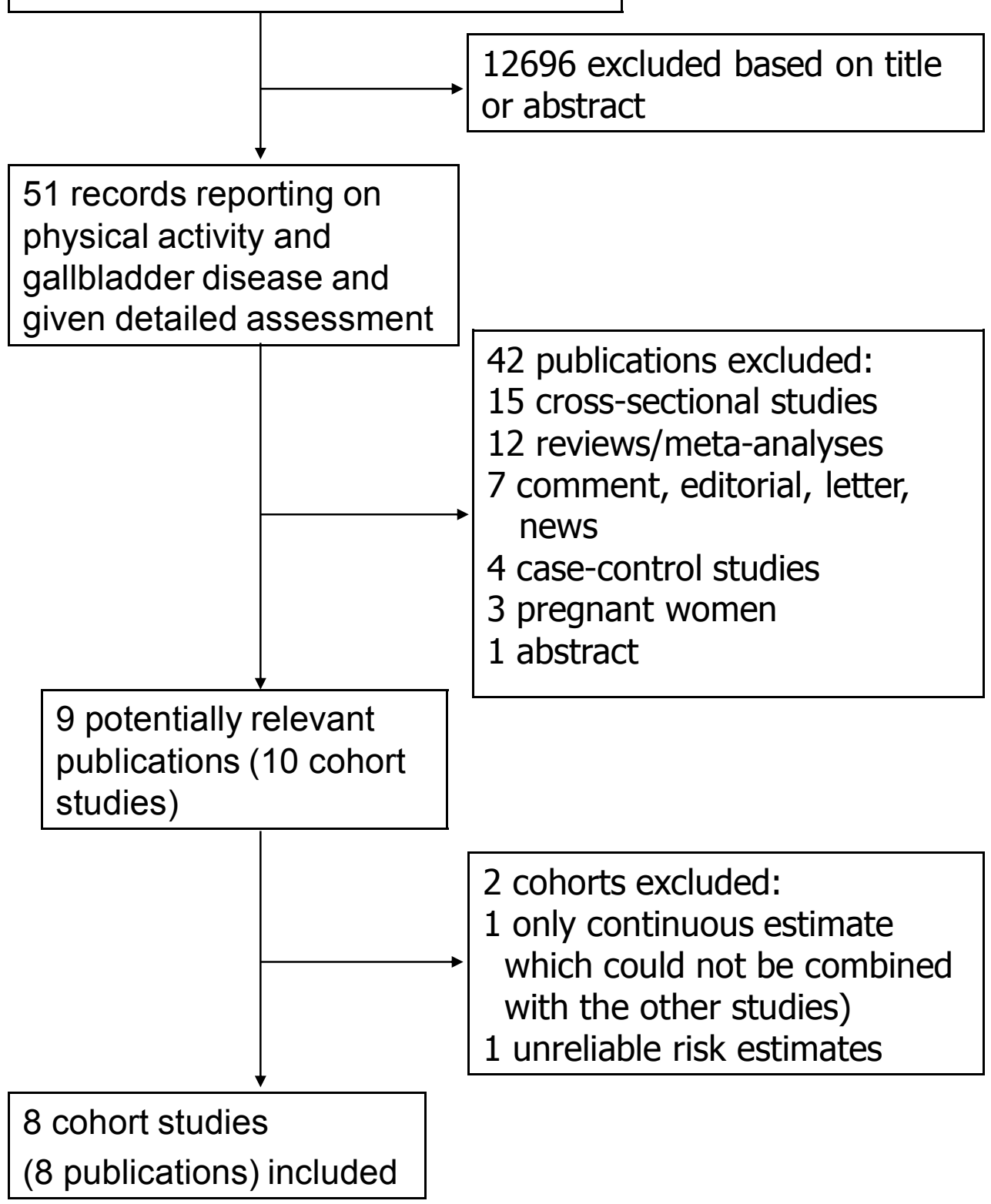


Figure 2. Physical activity and gallbladder disease, highest versus lowest comparison

Study

Banim, 2010

Williams, 2008

Storti, 2005

Boland, 2002

Leitzmann, 1999

Leitzmann, 1998

Sahi, 1998

Kato, 1992

Overall

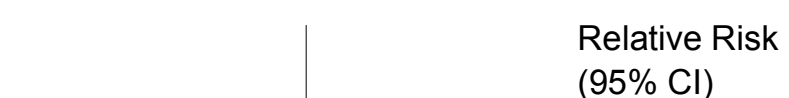

$(95 \% \mathrm{Cl})$

$0.70(0.48,1.01)$

$0.73(0.49,1.10)$

$0.63(0.44,0.90)$

$0.76(0.56,1.02)$

$0.79(0.71,0.89)$

$0.72(0.58,0.90)$

$0.72(0.46,1.13)$

$0.70(0.60,1.00)$

$0.75(0.69,0.81)$

\section{5}

.25


1 Physical activity and the risk of gallbladder disease: a

2 systematic review and meta-analysis of cohort studies.

4 Short title: Physical activity and gallbladder disease

5 Manuscript type: Review

6 Keywords: Exercise, gallstones, cohort studies.

7 Word count abstract: 200

8 Word count text: 2721

9

10

11

12 
Physical activity and gallbladder disease

\section{Abstract}

2 Background: Physical activity has been hypothesized to reduce the risk of gallbladder

3 disease (gallstones, cholecystitis, cholecystectomy), however, results from epidemiological

4 studies have not always shown statistically significant associations. We conducted a

5 systematic review and meta-analysis to clarify the presence and strength of an association

6 between physical activity and gallbladder disease risk.

7

8 Methods: PubMed and Embase databases were searched for studies of physical activity and

9 gallbladder disease up to $9^{\text {th }}$ of January 2015. Prospective studies reporting relative risk (RR)

10 estimates and 95\% confidence intervals (CIs) of gallbladder disease associated with physical

11 activity were included. Summary RRs were estimated using a random effects model.

13 Results: Eight studies including 6958 cases and 218204 participants were included. The

14 summary RR for the highest versus the lowest level of physical activity was 0.75 (95\% CI:

$150.69-0.81, \mathrm{n}=8$ ) and there was no evidence of heterogeneity, $\mathrm{I}^{2}=0 \%$ ). In the dose-response

16 analysis the summary relative risk per 20 MET-hours of activity was 0.85 (95\% CI: 0.80-

$\left.170.90, \mathrm{I}^{2}=0 \%, \mathrm{n}=2\right)$ for leisure-time physical activity, $0.83\left(95 \% \mathrm{CI}: 0.76-0.90, \mathrm{I}^{2}=0 \%, \mathrm{n}=2\right)$

18 for vigorous physical activity, and $0.86\left(95 \% \mathrm{CI}: 0.76-0.98, \mathrm{I}^{2}=0 \%, \mathrm{n}=2\right)$ for non-vigorous

19 physical activity.

20

21 Conclusion: Our analysis confirms a protective effect of physical activity on risk of

22 gallbladder disease. 


\section{Introduction}

2 Gallbladder disease (including gallstones and cholecystitis) is an important cause of

3 morbidity in European and North-American populations. Of digestive diseases that require

4 hospitalization gallstones are the most frequent and costly; the economic costs of hospital

5 treatment of gallstones is over 5 billion US dollar per year. ${ }^{1}$ In the United Kingdom 49000

6 cholecystectomies are conducted every year. ${ }^{2}$ The prevalence of gallstones varies

7 substantially in sonographic surveys around the world, with prevalence rates ranging from 3-

$86 \%$ in Asian countries, 5-10\% in African countries, and 10-30\% in most European and North-

9 American populations. ${ }^{3}$ In addition, secular trend studies suggest an increase in gallstone

10 prevalence over time in Asian countries parallel to changes in lifestyle. ${ }^{4}$ These observations

11 suggest that modifiable risk factors are important in the etiology of gallstones or gallbladder

12 disease.

13 Several studies have shown that greater body fat is a strong risk factor for gallbladder

14 disease with 2-5 fold increases in risk among obese compared to lean subjects. ${ }^{5-7}$ Physical

15 activity is known to reduce the risk of metabolic abnormalities such as insulin resistance, ${ }^{8,9}$

16 elevated triglycerides, ${ }^{10}$ low levels of high-density lipoprotein cholesterol, ${ }^{10}$ diabetes, ${ }^{11}$ and

17 general and abdominal obesity, ${ }^{12-14}$ which are important risk factors for gallbladder disease. ${ }^{15-}$

$18{ }^{19}$ Several epidemiological studies have shown inverse associations between physical activity

19 and gallbladder disease, ${ }^{20-23}$ although some showed statistically non-significant

20 associations, ${ }^{19,24-26}$ but it is not known what level of physical activity is needed to

21 significantly reduce the risk. We conducted a systematic review and meta-analysis of cohort

22 studies of physical activity and risk of gallbladder disease with the aim of clarifying the

23 presence and strength of the association, the dose-response relationship and how much of the

24 association may be explained by reductions in body fatness. 
Physical activity and gallbladder disease

\section{Methods}

2

3 Search strategy

$4 \quad$ We searched the Pubmed and Embase databases from inception (1966 and 1947,

5 respectively) up to $9^{\text {th }}$ of January 2015 for eligible studies. As part of a larger project on risk

6 factors for gallbladder disease we used broad search terms in the PubMed search: (body mass

7 index OR BMI OR overweight OR obesity OR anthropometry OR fatness OR body fatness

8 OR abdominal fatness OR abdominal obesity OR waist circumference OR waist-to-hip ratio

9 OR physical activity OR exercise OR sports OR walking OR biking OR running OR fitness

10 OR exercise test OR inactivity OR diabetes OR smoking OR tobacco OR risk factor OR risk

11 factors) AND (gallstones OR gallbladder disease OR cholelithiasis OR cholecystectomy OR

12 cholecystitis). This search strategy was modified and adapted for the Embase search

13 (Supplementary text). We followed standard criteria for conducting and reporting meta-

14 analyses. $^{27}$ In addition, we searched the reference lists of the identified publications for

15 further studies. Study quality was assessed using the Newcastle-Ottawa scale. ${ }^{28}$

\section{Inclusion criteria and study selection}

18 We included published prospective cohort studies (which are less prone to biases than case-

19 control and cross-sectional studies) that investigated the association between physical activity

20 and the risk of gallstones, gallbladder disease or cholecystectomies. Estimates of the relative

21 risk with the $95 \%$ CIs had to be available in the publication. For the dose-response meta-

22 analysis a quantitative measure of the physical activity level had to be provided. Cross-

23 sectional and case-control studies, reviews, meta-analyses, comments, editorials, letters, news

24 articles, studies in pregnant women, and abstract and grey literature was excluded. 
1 Supplementary Table 1 shows the excluded studies and reasons for exclusions. One of the

2 included articles ${ }^{23}$ reported results from two studies, but we only included results from one of

3 these (Study of Osteoporotic Fractures) because we considered the risk estimates from the

4 other study (Walking Women Follow-up Study) to be unreliable given the low number of

5 cases and participants, with RRs of 1.00 (reference, 0 cases/42 participants), 1.08 (95\% CI:

$6 \quad 0.96-1.22,3$ cases/41 participants), and 1.13 (95\% CI: $1.01-1.28,6$ cases/41 participants) for

7 tertiles 3,2 , and 1 , respectively.

\section{Data extraction}

10 The following data were extracted from each study: The first author's last name, publication

11 year, country where the study was conducted, study period, sample size, number of

12 cases/participants, physical activity type, level of activity, relative risks and 95\% confidence

13 intervals for the highest versus the lowest level of physical activity and variables adjusted for

14 in the analysis.

\section{Statistical methods}

17 We calculated summary relative risks for the highest versus the lowest level of physical 18 activity using the random-effects model by DerSimonian and Laird, ${ }^{29}$ which takes into 19 account both within and between study variation (heterogeneity). The average of the natural 20 logarithm of the relative risks was estimated and the relative risk from each study was 21 weighted by the inverse of its variance. For one study ${ }^{26}$ which only reported a continuous 22 estimate per $\mathrm{km}$ running (a different measure than the other studies which were reported in 23 MET-hours per week) and which therefore could not be included in the dose-response 24 analysis, we converted the continuous estimate to an estimate that could be used for the high 25 vs. low meta-analysis by multiplying the $\ln (\mathrm{RR})$ and $\ln (\mathrm{CI})$ by 9 , and back-transforming the 
1 risk estimate to non-logarithmic scale. We chose to multiply by 9 because the cut-offs for

2 increasing quintiles were $<2,2-4,4-6,6-8, \geq 8 \mathrm{~km} /$ day (these were provided in a figure, but

3 categorical risk estimates were not reported), which would give midpoints in the lowest

4 category and the highest category of 1 and $9 \mathrm{~km} /$ day, respectively, assuming the open-ended

5 intervals to have the same distance as the adjacent intervals.

6 To investigate whether specific levels of physical activity were associated with

7 gallbladder disease, we used the method described by Greenland and Longnecker ${ }^{30}$ to

8 conduct dose-response analyses by computing study-specific slopes (linear trends) and 95\%

9 confidence intervals from the natural $\log$ of the relative risks and confidence intervals across

10 categories of physical activity level. The median or mean level of physical activity in each

11 category of physical activity was assigned to the corresponding relative risk for each study.

12 For studies that reported the physical activity level by ranges of activity, we estimated the

13 midpoint for each category by calculating the average of the upper and lower boundaries. We

14 conducted separate dose-response analyses for studies that reported amounts of physical

15 activity in metabolic equivalent task (MET)-hours or kcal of energy expenditure. The MET is

16 an index of the intensity of physical activity and is defined as the caloric expenditure per

17 kilogram of body weight per hour of activity divided by the equivalent per hour at rest. One

18 MET is considered equal to the energy cost of a person during quiet sitting, while walking at

19 a slow pace has a MET value of 2, and jogging and bicycling have MET values of 7-8. MET-

20 hours are the number of hours engaged in each activity multiplied with the MET value of that

21 activity, which are summed across different types of activities. Studies that did not quantify

22 the physical activity level were excluded from the dose-response analysis. We assessed a

23 potential nonlinear dose-response relationship between physical activity and gallbladder

24 disease using fractional polynomial models. ${ }^{31}$ We determined the best fitting second order

25 fractional polynomial regression model, defined as the one with the lowest deviance. A 
2

3

4

5

6

7

8

9

10

1 likelihood ratio test was used to assess the difference between the nonlinear and linear models

2 to test for nonlinearity. ${ }^{32}$

3 Heterogeneity between studies was evaluated using Q and $\mathrm{I}^{2}$ statistics. ${ }^{33} \mathrm{I}^{2}$ is the 4 proportion of total variation contributed by between-study variation rather than chance. $\mathrm{I}^{2}$ 5 values of $25 \%, 50 \%$ and $75 \%$ indicate low, moderate and high heterogeneity, respectively.

6 We conducted main analyses and subgroup analyses by study characteristics such as gender,

7 type of outcome (gallstones, gallbladder disease and cholecystectomy), number of cases, 8 geographic location and adjustment for confounding factors. Study quality was assessed

9 using the Newcastle-Ottawa scale which rates the studies from 0 to 9 by assessing the 10 selection, comparability and outcome of each study. ${ }^{28}$

by inspection of funnel plots. The statistical analyses were conducted using the software package Stata, version 10.1 software (StataCorp, Texas, US). 
Physical activity and gallbladder disease

\section{Results}

2

3 We identified 8 cohort studies that could be included in the analysis of physical activity and

4 gallbladder disease ${ }^{19-26}$, with a total of 6958 cases and 218204 participants (Figure 1, Table

5 1). Seven of the studies were from North-America and one was from Europe.

6

$7 \quad$ High versus low analysis

8 Eight cohort studies ${ }^{19-26}$ investigated the association between physical activity and

9 gallbladder disease risk (Table 1). The summary relative risk for high versus low physical

10 activity was 0.75 (95\% CI: $0.69-0.81)$ with no evidence of heterogeneity, $\mathrm{I}^{2}=0 \%$,

11 pheterogeneity $=0.94$ (Figure 2). There was some evidence of publication bias with Egger's test,

$12 \mathrm{p}=0.02$, but not with Begg's test, $\mathrm{p}=0.90$ (Supplementary Figure 1), but this disappeared

13 when we restricted the analysis to studies with $\geq 500$ cases, $p=0.14$, and the results were

14 similar, showing a summary RR of 0.76 (95\% CI: $0.69-0.84, \mathrm{I}^{2}=0 \%$, pheterogeneity $\left.=0.62\right)$.

Dose-response analyses

17 Only two cohort studies could be included in the dose-response analysis of leisure-time

18 physical activity in MET-hours per week. ${ }^{21,22}$ The summary relative risk was 0.85 (95\% CI:

$190.80-0.90, \mathrm{I}^{2}=0 \%$, pheterogeneity $=0.84$ ) per 20 MET-hours per week (Supplementary Figure 2a).

20 When BMI was omitted from the multivariate adjustment the summary RR was 0.78 (95\%

21 CI: $0.73-0.83, I^{2}=0 \%$, pheterogeneity $=0.54$ ) (Figure $\left.2 a\right)$. There was no evidence of a nonlinear

22 association between leisure-time physical activity and gallbladder disease, $p_{\text {nonlinearity }}=0.28$

23 (Figure 2b, Supplementary Table 2). Two cohort studies ${ }^{23,24}$ could be included in the dose-

24 response analysis of energy expended on physical activity per week (kcal per week). The 
1 summary relative risk per $1000 \mathrm{kcal} /$ week was 0.93 (95\% CI: $0.74-1.16, \mathrm{I}^{2}=88.7 \%$,

$\left.2 \quad p_{\text {heterogeneity }}=0.003\right)($ Supplementary Figure 3).

3

$4 \quad$ Vigorous and non-vigorous physical activity

5 Only two cohort studies could be included in the dose-response analysis of vigorous (defined

6 as activity that required 6 or more METs per hour) and non-vigorous (defined as activity that

7 required less than 6 METs per hour, including light and moderate activity) physical

8 activity. $^{21,22}$ The summary RR per 20 MET-hours per week was 0.83 (95\% CI: 0.76-0.90,

$9 \mathrm{I}^{2}=0 \%$, pheterogeneity $\left.=0.85\right)$ for vigorous physical activity and it was 0.86 (95\% CI: $0.76-0.98$,

$10 \mathrm{I}^{2}=0 \%, p_{\text {heterogeneity }}=0.45$ ) for non-vigorous physical activity (Supplementary Figure $4 \mathrm{a}$,

11 Supplementary Figure 5a). When BMI was omitted from the multivariate adjustment the

12 respective summary RRs were $0.76\left(95 \% \mathrm{CI}: 0.70-0.83, \mathrm{I}^{2}=0 \%\right.$, pheterogeneity $\left.=0.94\right)$ and 0.78

$13\left(95 \%\right.$ CI: $\left.0.65-0.93, I^{2}=36 \%, p_{\text {heterogeneity }}=0.21\right)$ (Supplementary Figure 4a, Supplementary

14 Figure 5a). There was evidence of a nonlinear association between vigorous and non-

15 vigorous physical activity and gallbladder disease, $p_{\text {nonlinearity }}=0.009$ and $p_{\text {nonlinearity }}=<0.0001$,

16 respectively, with a steeper inverse association at higher levels of vigorous physical activity,

17 while the association for non-vigorous physical activity was less pronounced at higher levels

18 of activity (Supplementary Figure 4b, Supplementary Figure 5b, Supplementary Table 2).

\section{Subgroup and sensitivity analyses}

21 Relations of physical activity to gallbladder disease were inverse in all strata of subgroup 22 analyses, defined by gender, duration of follow-up, geographic location, type of outcome, 23 number of cases, study quality and adjustment for confounding factors (Table 2). With meta24 regression analyses there was no evidence that the results differed between these subgroups 25 (Table 2). Mean and median study quality scores were 6.13 and 6.5, respectively. When we 
Physical activity and gallbladder disease

32

33

34

35

36

1 conducted sensitivity analyses removing one study at a time, the size of the summary RR

2 ranged from 0.71 (95\% CI: $0.63-0.80)$ when excluding the study by Leitzmann et $\mathrm{al}^{22}$ to 0.76

3 (95\% CI: 0.70-0.82) when excluding the study by Storti et al. ${ }^{23}$

\section{4}

5

6

7 
Discussion

To our knowledge this is the first meta-analysis of observational studies of physical

3 activity and risk of gallbladder disease. Our results confirm an inverse association between

4 higher levels of physical activity and gallbladder disease. There was a $25 \%$ relative risk

5 reduction for participants with the highest versus the lowest physical activity level. Both

$6 \quad$ vigorous and non-vigorous physical activity was associated with reduced risk.

The present systematic review and meta-analysis has some limitations that need to be

8 discussed. The number of studies included was modest and few studies could be included in

9 the dose-response analysis because the physical activity level was not quantified or it was

10 quantified in a manner that was not possible to combine with the remaining studies. Further

11 studies should aim to report results in a manner that can be included in future updated dose-

12 response meta-analyses (for example in MET-hrs per week). Our analysis suggests that both

13 vigorous and non-vigorous physical activity reduces risk of gallbladder disease, although the

14 dose-response relationship appeared to be stronger for vigorous physical activity than for

15 non-vigorous physical activity. This might at least partly be due to the greater range of

16 vigorous physical activity than of non-vigorous activity. However, further studies are needed

17 as the number of studies on subtypes of physical activity was low. Many of the included

18 studies adjusted for important confounding factors and in subgroup analyses, we did not find

19 evidence that the results differed when stratified by adjustment for confounding factors. We

20 cannot, however, exclude the possibility that residual confounding by other unidentified risk

21 factors could have partly affected the results. Physical activity was assessed using self-

22 reported questionnaires and therefore measurement error in the exposure variable is likely to

23 have occurred. Two studies made corrections for measurement error using data from their

24 validation studies. ${ }^{21,22}$ One of the studies reported a RR of 0.81 (95\% CI: $\left.0.73-0.89\right)$ per 25

25 MET-hours per week of moderate intensity exercise in an uncorrected model, and 0.64 (95\% 
Physical activity and gallbladder disease

1 CI: 0.49-0.86) in a model corrected for measurement error, ${ }^{21}$ and another study reported a RR

2 of 0.85 (95\% CI: 0.80-0.90) per 25 MET-hours per week in an uncorrected model and 0.58

3 (95\% CI: 0.43-0.77) in a model corrected for measurement error, ${ }^{22}$ suggesting substantial

4 underestimation of the RR due to measurement errors. In addition, because of the limited

5 number of studies we chose to analyse leisure-time physical activity together with total

6 physical activity. However, the magnitude of the risk estimates were similar in stratified

7 analyses, and there was no heterogeneity between these subgroups. There were some

8 differences in the definition of the outcome as well. Some studies investigated only

9 gallstones, while others investigated gallbladder disease which included both gallstones and

10 inflammation of the gallbladder (cholecystitis), and in one study the outcome was surgical

11 removal of the gallbladder (cholecystectomy), while no cohort studies reported on

12 cholecystitis only. However, risk estimates where similar when stratified by type of outcome

13 and there was no evidence of heterogeneity between these subgroups. Because most of the

14 studies were from North America and included mainly Caucasian subjects, further studies

15 among other ethnicities and in other geographic locations are needed to clarify if the findings

16 also apply to other populations. One last limitation is that the study selection was done by one

17 author and not in duplicate.

18 Several biological mechanisms could explain a protective effect of physical activity

19 on risk of gallbladder disease. Physical activity has been established to protect against general

20 and abdominal overweight and obesity ${ }^{12-14}$ and diabetes ${ }^{36}$ which are strongly associated with

21 risk of gallbladder disease..$^{5,7,16,17}$ However, in this meta-analysis most studies adjusted for

22 BMI and several studies also adjusted for diabetes and the results persisted in these subgroup

23 analyses. In two studies ${ }^{21,22}$ that provided risk estimates both adjusted and not adjusted for

24 BMI the inverse association was slightly more pronounced when BMI was omitted from the

25 multivariable models compared to when adjusted for BMI (RR of 0.78 vs. 0.85 per 25 MET- 
1 hours/week for leisure-time activity), suggesting that reduced adiposity may explain

2 approximately $30-35 \%$ of the reduction in risk observed with physical activity. However, a

3 clinically relevant reduction in risk was observed even with adjustment for BMI and other

4 mechanisms are therefore likely to be involved in explaining the beneficial effects of physical

5 activity on gallbladder disease. Physical activity has been shown to reduce levels of total

6 cholesterol, ${ }^{10}$ triglycerides, ${ }^{10}$ and insulin resistance ${ }^{8,9}$ and this may further contribute to

7 reduced gallbladder disease risk. In addition, physical activity may influence gallbladder

8 disease risk by modulating gallbladder motility. ${ }^{37}$ Hypomotility of the gallbladder leads to

9 incomplete emptying of the gallbladder which promotes bile stasis and crystal formation

10 leading to gallstones. ${ }^{38}$ Physical activity may also increase vagal tone which can stimulate

11 gallbladder emptying. ${ }^{39}$

12 Strengths of this analysis include the large sample size which provides sufficient

13 statistical power to detect modest associations, and comprehensive subgroup, sensitivity and

14 dose-response analyses, as well as moderate to high study quality. Because this analysis only

15 included cohort studies recall bias is not likely to explain the findings and there is less

16 possibility for selection bias. When stratified by gender, similar reductions in risk were

17 observed for men and women.

18 In conclusion, the results from this systematic review and meta-analysis confirm that

19 a high level of physical activity reduces the risk of gallbladder disease. This adds to the many

20 health benefits that have already been documented for physical activity. Future studies should

21 attempt to further define the dose-response relationship between physical activity and specific

22 types of physical activity and gallbladder disease risk and additional studies in non-American

23 populations are warranted. 
1 Acknowledgement/Contribution: DA designed the research, conducted the literature search

2 and analyses and wrote the first draft of the paper. All authors interpreted the data, revised the

3 subsequent drafts for important intellectual content, read and approved the final manuscript.

4

5 Conflict of interest: The authors declare that there is no duality of interest associated with

6 this manuscript.

7

8 Funding source: This work was funded by "Liaison Committee between the Central Norway

9 Regional Health Authority (RHA) and the Norwegian University of Science and Technology

10 (NTNU)".

11

12

13

14

15

16

17

18

19

20 
(1) Sandler RS, Everhart JE, Donowitz M et al. The burden of selected digestive diseases in the United States. Gastroenterology. 2002;122:1500-1511. [PubMed: 11984534]

(2) Sanders G, Kingsnorth AN. Gallstones. BMJ. 2007;335:295-299. [PubMed: 17690370]

(4) Park YH, Park SJ, Jang JY et al. Changing patterns of gallstone disease in Korea. World J

(5) Stampfer MJ, Maclure KM, Colditz GA, Manson JE, Willett WC. Risk of symptomatic gallstones in women with severe obesity. Am J Clin Nutr. 1992;55:652-658. [PubMed: 1550039]

(6) Liu B, Balkwill A, Spencer E, Beral V. Relationship between body mass index and length of hospital stay for gallbladder disease. J Public Health (Oxf). 2008;30:161-166. [PubMed: $18308742]$

(7) Aune D, Norat T, Vatten LJ. Body mass index, abdominal fatness and the risk of gallbladder disease. Eur J Epidemiol. 2015;30:1009-1019. [PubMed: 26374741]

(8) Riserus U, Arnlov J, Berglund L. Long-term predictors of insulin resistance: role of lifestyle and metabolic factors in middle-aged men. Diabetes Care. 2007;30:2928-2933. [PubMed: 17644620] 
Physical activity and gallbladder disease

1

2

3

4

5

6

7

8

9

10

11

12

13

14

15

16

17

18

19

20

(9) Assah FK, Brage S, Ekelund U, Wareham NJ. The association of intensity and overall level of physical activity energy expenditure with a marker of insulin resistance. Diabetologia. 2008;51:1399-1407. [PubMed: 18488189]

(10) Kelley GA, Kelley KS, Tran ZV. Aerobic exercise and lipids and lipoproteins in women: a meta-analysis of randomized controlled trials. J Womens Health (Larchmt). 2004;13:11481164. [PubMed: 15650348]

(11) Jeon CY, Lokken RP, Hu FB, van Dam RM. Physical activity of moderate intensity and risk of type 2 diabetes: a systematic review. Diabetes Care. 2007;30:744-752. [PubMed: $17327354]$

(12) Mozaffarian D, Hao T, Rimm EB, Willett WC, Hu FB. Changes in diet and lifestyle and longterm weight gain in women and men. $N$ Engl J Med. 2011;364:2392-2404. [PubMed: 21696306]

(13) Ekelund U, Besson H, Luan J et al. Physical activity and gain in abdominal adiposity and body weight: prospective cohort study in 288,498 men and women. Am J Clin Nutr. 2011;93:826-835. [PubMed: 21346093]

(14) Davidson LE, Tucker L, Peterson T. Physical activity changes predict abdominal fat change in midlife women. J Phys Act Health. 2010;7:316-322. [PubMed: 20551487]

(15) Liu CM, Tung TH, Tsai ST et al. Serum insulin, insulin resistance, beta-cell dysfunction, and gallstone disease among type 2 diabetics in Chinese population: a community-based study in Kinmen, Taiwan. World J Gastroenterol. 2005;11:7159-7164. [PubMed: 16437664] 
1

2

3

4

5

6

7

8

9

10

11

12

13

14

15

16

17

18

19

20

(16) Jamal MM, Yoon EJ, Vega KJ, Hashemzadeh M, Chang KJ. Diabetes mellitus as a risk factor for gastrointestinal cancer among American veterans. World J Gastroenterol. 2009;15:52745278. [PubMed: 19908334]

(17) Aune D, Vatten LJ. Diabetes mellitus and the risk of gallbladder disease: A systematic review and meta-analysis of prospective studies. J Diabetes Complications. 2015. doi: 10.1016/j.jdiacomp.2015.11.012. [Epub ahead of print] [PubMed: 26684168]

(18) Tsai CJ, Leitzmann MF, Willett WC, Giovannucci EL. Prospective study of abdominal adiposity and gallstone disease in US men. Am J Clin Nutr. 2004;80:38-44. [PubMed: $15213025]$

(19) Banim PJ, Luben RN, Wareham NJ, Sharp SJ, Khaw KT, Hart AR. Physical activity reduces the risk of symptomatic gallstones: a prospective cohort study. Eur J Gastroenterol Hepatol. 2010;22:983-988. [PubMed: 20130468]

(20) Kato I, Nomura A, Stemmermann GN, Chyou PH. Prospective study of clinical gallbladder disease and its association with obesity, physical activity, and other factors. Dig Dis Sci. 1992;37:784-790. [PubMed: 1563324]

(21) Leitzmann MF, Giovannucci EL, Rimm EB et al. The relation of physical activity to risk for symptomatic gallstone disease in men. Ann Intern Med. 1998;128:417-425. [PubMed: 1563324]

(22) Leitzmann MF, Rimm EB, Willett WC et al. Recreational physical activity and the risk of cholecystectomy in women. N Engl J Med. 1999;341:777-784. [PubMed 10477775] 
Physical activity and gallbladder disease

1

2

3

4

5

6

7

8

9

10

11

12

13

14

15

16

17

18

19

20

(23) Storti KL, Brach JS, FitzGerald SJ, Zmuda JM, Cauley JA, Kriska AM. Physical activity and decreased risk of clinical gallstone disease among post-menopausal women. Prev Med. 2005;41:772-777. [PubMed: 16038966]

(24) Sahi T, Paffenbarger RS, Jr., Hsieh CC, Lee IM. Body mass index, cigarette smoking, and other characteristics as predictors of self-reported, physician-diagnosed gallbladder disease in male college alumni. Am J Epidemiol. 1998;147:644-651. [PubMed: 9554603]

(25) Boland LL, Folsom AR, Rosamond WD. Hyperinsulinemia, dyslipidemia, and obesity as risk factors for hospitalized gallbladder disease. A prospective study. Ann Epidemiol. 2002;12:131-140. [PubMed: 11880221]

(26) Williams PT. Independent effects of cardiorespiratory fitness, vigorous physical activity, and body mass index on clinical gallbladder disease risk. Am J Gastroenterol. 2008;103:22392247. [PubMed 18637096]

(27) Stroup DF, Berlin JA, Morton SC et al. Meta-analysis of observational studies in epidemiology: a proposal for reporting. Meta-analysis Of Observational Studies in Epidemiology (MOOSE) group. JAMA. 2000;283:2008-2012. [PubMed: 10789670]

(28) Wells G, Shea B, O'Connell D. et al. The Newcastle-Ottawa Scale (NOS) for assessing the quality of nonrandomised studies in meta-analyses. $\underline{h t p: / / w w w}$ ohri.ca/programs/clinical_epidemiology/oxford.asp, Accessed 13.08.2014.

(29) DerSimonian R, Laird N. Meta-analysis in clinical trials. Control Clin Trials. 1986;7:177188. [PubMed: 3802833] 
1

2

3

4

5

6

7

8

9

10

(30) Greenland S, Longnecker MP. Methods for trend estimation from summarized dose-response data, with applications to meta-analysis. Am J Epidemiol. 1992;135:1301-1309. [PubMed: 1626547]

(31) Royston P. A strategy for modelling the effect of a continuous covariate in medicine and epidemiology. Stat Med. 2000;19:1831-1847. [PubMed: 10867674]

(32) Bagnardi V, Zambon A, Quatto P, Corrao G. Flexible meta-regression functions for modeling aggregate dose-response data, with an application to alcohol and mortality. Am J Epidemiol. 2004;159:1077-1086. [PubMed: 15155292]

(33) Higgins JP, Thompson SG. Quantifying heterogeneity in a meta-analysis. Stat Med. 2002;21:1539-1558. [PubMed: 12111919]

(34) Egger M, Davey SG, Schneider M, Minder C. Bias in meta-analysis detected by a simple, graphical test. BMJ. 1997;315:629-634. [PubMed: 9310563]

(35) Begg CB, Mazumdar M. Operating characteristics of a rank correlation test for publication bias. Biometrics. 1994;50:1088-1101. [PubMed: 7786990]

(36) Aune D, Norat T, Leitzmann M, Tonstad S, Vatten LJ. Physical activity and the risk of type 2 diabetes: a systematic review and dose-response meta-analysis. Eur J Epidemiol. 2015;30:529-542. [PubMed: 26092138]

(37) Sari R, Balci N, Balci MK. Effects of exercise on gallbladder volume and motility in obese women. J Clin Ultrasound. 2005;33:218-222. [PubMed: 16047386] 
Physical activity and gallbladder disease

1

2

3

4

5

6

7

8

9

10

(38) Bowen JC, Brenner HI, Ferrante WA, Maule WF. Gallstone disease. Pathophysiology, epidemiology, natural history, and treatment options. Med Clin North Am. 1992;76:11431157. [PubMed: 1518331]

(39) Krotkiewski M, Bjorntorp P, Holm G et al. Effects of physical training on insulin, connecting peptide (C-peptide), gastric inhibitory polypeptide (GIP) and pancreatic polypeptide (PP) levels in obese subjects. Int J Obes. 1984;8:193-199. [PubMed: 6378815] 
Table 1. Prospective studies of physical activity and gallbladder disease

\begin{tabular}{|c|c|c|c|c|c|c|}
\hline $\begin{array}{l}\text { Number of } \\
\text { participants, } \\
\text { number of } \\
\text { cases }\end{array}$ & $\begin{array}{l}\text { Study } \\
\text { period, } \\
\text { duration of } \\
\text { follow-up }\end{array}$ & $\begin{array}{l}\text { Study } \\
\text { quality } \\
\text { score }\end{array}$ & Physical activity exposure & Quantity of physical activity & $\begin{array}{l}\text { Relative risk } \\
(95 \% \\
\text { confidence } \\
\text { interval })\end{array}$ & Adjustment for confounders \\
\hline $\begin{array}{l}\text { 7831 Japanese } \\
\text { men, age } \geq 45- \\
65: 471 \\
\text { gallbladder } \\
\text { disease }\end{array}$ & $\begin{array}{l}1965-68- \\
1990\end{array}$ & 8 & Physical activity index (TPA) & $\geq 35.5$ vs. $<29.5 \mathrm{METs} / \mathrm{d}$ & $0.7(0.6-1.0)$ & $\begin{array}{l}\text { Age, pack-years of cigarettes, } \\
\text { alcohol, serum TG, serum uric } \\
\text { acid, serum glucose, DBP, height, } \\
\text { BMI, total calories }\end{array}$ \\
\hline $\begin{array}{l}16414 \text { men, } \\
\text { age } 32-74 \\
\text { years: } 268 \\
\text { gallbladder } \\
\text { disease cases }\end{array}$ & $\begin{array}{l}1962-1966- \\
1977, \text { up to } \\
15 \text { years } \\
\text { follow-up }\end{array}$ & 7 & Physical activity index (LTPA) & $\geq 3500$ vs. $<500 \mathrm{kcal} / \mathrm{wk}$ & $0.72(0.46-1.13)$ & $\begin{array}{l}\text { Age, calendar year, BMI, BMI } \\
\text { change between college and } \\
\text { 1962/66, smoking habit }\end{array}$ \\
\hline $\begin{array}{l}45813 \text { men, } \\
\text { age } 40-75 \\
\text { years: } 828 \\
\text { symptomatic } \\
\text { gallstone } \\
\text { cases }\end{array}$ & $\begin{array}{l}1986-1994 \\
8 \text { years } \\
\text { follow-up }\end{array}$ & 7 & $\begin{array}{l}\text { Total physical activity (LTPA) } \\
\text { Total physical activity (LTPA) } \\
\text { Total physical activity (LTPA), BMI adj. } \\
\text { Vigorous activity } \\
\text { Vigorous activity } \\
\text { Vigorous activity, BMI adj. } \\
\text { Nonvigorous activity } \\
\text { Nonvigorous activity } \\
\text { Nonvigorous activity, BMI adj. }\end{array}$ & $\begin{array}{l}\geq 32.6 \text { vs. } 0-2.7 \mathrm{MET}-\mathrm{hrs} / \mathrm{wk} \\
\text { Per } 25 \mathrm{MET}-\mathrm{hrs} / \mathrm{wk} \\
\geq 32.6 \text { vs. } 0-2.7 \mathrm{MET}-\mathrm{hrs} / \mathrm{wk} \\
\geq 25.1 \text { vs. } 0 \mathrm{MET}-\mathrm{hrs} / \mathrm{wk} \\
\text { Per } 25 \mathrm{MET}-\mathrm{hrs} / \mathrm{wk} \\
\geq 25.1 \text { vs. } 0 \mathrm{MET}-\mathrm{hrs} / \mathrm{wk} \\
\geq 11.4 \text { vs. } 0-0.8 \mathrm{MET}-\mathrm{hrs} / \mathrm{wk} \\
\text { Per } 25 \mathrm{MET}-\mathrm{hrs} / \mathrm{wk} \\
\geq 11.4 \text { vs. } 0-0.8 \mathrm{MET}-\mathrm{hrs} / \mathrm{wk}\end{array}$ & $\begin{array}{l}0.63(0.51-0.79) \\
0.81(0.73-0.89) \\
0.72(0.59-0.90) \\
0.58(0.45-0.75) \\
0.77(0.67-0.88) \\
0.65(0.50-0.85) \\
0.93(0.75-1.15) \\
0.87(0.73-1.03) \\
0.97(0.78-1.19)\end{array}$ & $\begin{array}{l}\text { Age, diabetes mellitus, smoking, } \\
\text { cholesterol-lowering drugs, } \\
\text { thiazide use, NSAIDS, alcohol, } \\
\text { dietary fiber, carbohydrates }\end{array}$ \\
\hline $\begin{array}{l}60290 \\
\text { women, age } \\
40-65 \text { years: } \\
3257 \\
\text { cholecystec- } \\
\text { tomies }\end{array}$ & $\begin{array}{l}1986-1996, \\
10 \text { years } \\
\text { follow-up }\end{array}$ & 7 & $\begin{array}{l}\text { Physical activity (LTPA) } \\
\text { Physical activity, BMI/weight change-adj. } \\
\text { Physical activity, BMI/weight change-adj. } \\
\text { Vigorous physical activity } \\
\text { Vigorous physical activity, BMI/weight } \\
\text { change-adj. } \\
\text { Vigorous physical activity, BMI/weight } \\
\text { change-adj. } \\
\text { Nonvigorous physical activity } \\
\text { Nonvigorous physical activity, BMI/weight }\end{array}$ & $\begin{array}{l}\geq 22.1 \text { vs. } 0-1.6 \mathrm{MET}-\mathrm{hrs} / \mathrm{wk} \\
\geq 22.1 \text { vs. } 0-1.6 \mathrm{MET}-\mathrm{hrs} / \mathrm{wk} \\
\text { Per } 25 \mathrm{MET}-\mathrm{hrs} / \mathrm{wk} \\
\geq 16 \text { vs. } 0 \mathrm{MET}-\mathrm{hrs} / \mathrm{wk} \\
\geq 16 \text { vs. } 0 \mathrm{MET}-\mathrm{hrs} / \mathrm{wk} \\
\text { Per } 10 \mathrm{MET}-\mathrm{hrs} / \mathrm{wk} \\
\geq 10.8 \text { vs. } 0-0.8 \mathrm{MET}-\mathrm{hrs} / \mathrm{wk} \\
\geq 10.8 \text { vs. } 0-0.8 \mathrm{MET}-\mathrm{hrs} / \mathrm{wk}\end{array}$ & $\begin{array}{l}0.69(0.61-0.78) \\
0.79(0.71-0.89) \\
0.85(0.80-0.90) \\
0.75(0.66-0.85) \\
0.82(0.73-0.93) \\
0.89(0.86-0.94) \\
0.86(0.77-0.98) \\
0.95(0.84-1.06)\end{array}$ & $\begin{array}{l}\text { Age, parity, OC use, use of HRT, } \\
\text { diabetes mellitus, pack-years of } \\
\text { smoking, use of cholesterol- } \\
\text { lowering drugs, thiazide use, } \\
\text { NSAIDS, dietary fiber, } \\
\text { carbohydrates, alcohol, coffee }\end{array}$ \\
\hline
\end{tabular}


Physical activity and gallbladder disease

\begin{tabular}{|c|c|c|c|c|c|c|c|}
\hline $\begin{array}{l}5 \\
6 \\
7\end{array}$ & & & & $\begin{array}{l}\text { change-adj. } \\
\text { Nonvigorous physical activity, BMI/weight } \\
\text { change-adj. }\end{array}$ & Per 10 MET-hrs/wk & $0.91(0.87-0.96)$ & \\
\hline $\begin{array}{l}8 \text { Boland LL } \\
9 \text { et al, } \\
102002, \\
11 \text { USA } \\
12 \\
13\end{array}$ & $\begin{array}{l}12773 \text { men } \\
\text { and women, } \\
\text { age } 45-64 \\
\text { years: } 397 \\
\text { gallbladder } \\
\text { disease }\end{array}$ & $\begin{array}{l}1987-1989- \\
1996,8.2 \\
\text { years } \\
\text { follow-up }\end{array}$ & 6 & \begin{tabular}{|l|} 
Sport index (LTPA), men \\
Sport index (LTPA), women
\end{tabular} & $\begin{array}{l}\geq 3.25 \text { vs. } \leq 1.75 \\
\geq 3.25 \text { vs. } \leq 1.75\end{array}$ & $\begin{array}{l}0.81(0.5-1.3) \\
0.72(0.5-1.1)\end{array}$ & Age \\
\hline $\begin{array}{l}1 \text { \$torti KL } \\
1 \text { ftal, } 2005 \\
16^{\text {USA }} \\
17 \\
\end{array}$ & $\begin{array}{l}8010 \text { women, } \\
\text { age } \geq 65 \text { years: } \\
1281 \text { gallstone } \\
\text { disease cases }\end{array}$ & $\begin{array}{l}\text { 1989-90- } \\
1992-1994, \\
\sim 3.5 \text { years } \\
\text { follow-up } \\
\end{array}$ & 5 & $\begin{array}{l}\text { Physical activity (LTPA) } \\
\text { Physical activity (LTPA) } \\
\end{array}$ & $\begin{array}{l}\text { Per } 500 \mathrm{kcal} / \mathrm{wk} \\
>2244 \text { vs. }<512 \mathrm{kcal} / \mathrm{wk}\end{array}$ & $\begin{array}{l}0.94(0.90-0.98) \\
0.63(0.44-0.90)\end{array}$ & $\begin{array}{l}\text { Age, BMI, education, hormone use, } \\
\text { diabetes prevalence, smoking } \\
\text { status, alcohol use, parity }\end{array}$ \\
\hline $\begin{array}{l}18 \text { Villiams } \\
19 \text { PT et al, } \\
20 \text { 2008, } \\
21 \\
22 \\
23 \\
23 \\
24\end{array}$ & $\begin{array}{l}29110 \text { male } \\
\text { and } 11953 \\
\text { female } \\
\text { runners, mean } \\
\text { age } 45 / 39 \\
\text { years: } 166 \\
\text { gallbladder } \\
\text { disease cases }\end{array}$ & $\begin{array}{l}\text { 1991-94- } \\
2002,7.6 \\
\text { years } \\
\text { follow-up }\end{array}$ & 4 & $\begin{array}{l}\text { Physical activity (LTPA), men } \\
\text { Physical activity (LTPA), men, BMI adj. } \\
\text { Physical activity (LTPA), women } \\
\text { Physical activity (LTPA), women, BMI adj. }\end{array}$ & $\begin{array}{l}\text { Per km/d } \\
\text { Per km/d } \\
\text { Per km/d } \\
\text { Per km/d }\end{array}$ & $\begin{array}{l}0.93(0.87-0.98) \\
0.97(0.91-1.02) \\
0.91(0.84-0.97) \\
0.96(0.89-1.03)\end{array}$ & $\begin{array}{l}\text { Age, race, education, pack-yrs of } \\
\text { cigarette use, weekly intakes of } \\
\text { meat, fish, fruit, alcohol, parity }\end{array}$ \\
\hline $\begin{array}{l}25 \text { Banim PJR } \\
\text { 26t al, 2010, } \\
2 \text { Jnited } \\
26 \text { Kingdom } \\
29 \\
30\end{array}$ & $\begin{array}{l}25639 \text { men } \\
\text { and women, } \\
\text { age } 40-74 \\
\text { years: } 290 \\
\text { gallstone } \\
\text { cases }\end{array}$ & $\begin{array}{l}1993-1997 \\
-2007,14 \\
\text { years } \\
\text { follow-up }\end{array}$ & 8 & \begin{tabular}{|l} 
Physical activity (TPA), all \\
Physical activity (TPA), men \\
Physical activity (TPA), women
\end{tabular} & $\begin{array}{l}\text { Active vs. inactive } \\
\text { Active vs. inactive } \\
\text { Active vs. inactive }\end{array}$ & $\begin{array}{l}0.75(0.50-1.12) \\
0.81(0.43-1.51) \\
0.66(0.38-1.13)\end{array}$ & $\begin{array}{l}\text { Age, alcohol, BMI, sex } \\
\text { Women: also adjusted for parity } \\
\text { and HRT }\end{array}$ \\
\hline
\end{tabular}

$\mathrm{BMI}=$ Body mass index, $\mathrm{DM}=$ diabetes mellitus, $\mathrm{HRT}=$ hormone replacement therapy, $\mathrm{LTPA}=$ leisure-time physical activity, MUFA=monounsaturated fatty acids, $\mathrm{NA}=$ not available, NSAID=non-steroidal anti-inflammatory drugs, $\mathrm{OC}$ use $=$ oral contraceptive use, $\mathrm{SFA}=$ saturated fatty acids, $\mathrm{TPA}=$ total physical activity (leisure + occupational activity) 
Table 2. Subgroup analyses of physical activity and gallbladder disease

\begin{tabular}{|c|c|c|c|c|c|}
\hline & \multicolumn{5}{|c|}{ Physical activity and gallbladder disease } \\
\hline & $n$ & Relative risk $(95 \% \mathrm{CI})$ & $I^{2}(\%)$ & $P_{\mathrm{h}}{ }^{1}$ & $P_{\mathrm{h}}^{2}$ \\
\hline All studies & 8 & $0.75(0.69-0.81)$ & 0 & 0.94 & \\
\hline \multicolumn{6}{|l|}{ Gender } \\
\hline Men & 6 & $0.73(0.63-0.84)$ & 0 & 0.99 & 0.60 \\
\hline Women & 5 & $0.76(0.69-0.85)$ & 0 & 0.75 & \\
\hline \multicolumn{6}{|l|}{ Outcome } \\
\hline Gallstones & 3 & $0.70(0.59-0.82)$ & 0 & 0.82 & 0.24 \\
\hline Gallbladder disease & 4 & $0.73(0.62-0.85)$ & 0 & 0.98 & \\
\hline Cholecystectomy & 1 & $0.79(0.71-0.88)$ & & & \\
\hline \multicolumn{6}{|l|}{ Type of physical activity } \\
\hline Leisure-time physical activity & 6 & $0.76(0.70-0.83)$ & 0 & 0.87 & 0.51 \\
\hline Total physical activity & 2 & $0.70(0.57-0.86)$ & 0 & 1.00 & \\
\hline \multicolumn{6}{|l|}{ Geographic location } \\
\hline Europe & 1 & $0.70(0.48-1.02)$ & & & 0.72 \\
\hline America & 7 & $0.75(0.69-0.82)$ & 0 & 0.90 & \\
\hline \multicolumn{6}{|l|}{ Number of cases } \\
\hline Cases $<500$ & 4 & $0.72(0.62-0.85)$ & 0 & 0.98 & 0.43 \\
\hline Cases 500-<1000 & 2 & $0.72(0.59-0.88)$ & 28.4 & 0.24 & \\
\hline Cases $\geq 1000$ & 2 & $0.72(0.59-0.88)$ & 0 & 1.00 & \\
\hline \multicolumn{6}{|l|}{ Study quality } \\
\hline $0-3$ stars & 0 & & & & 0.16 \\
\hline 4-6 stars & 4 & $0.71(0.59-0.85)$ & 0 & 0.88 & \\
\hline $7-9$ stars & 4 & $0.76(0.69-0.83)$ & 0 & 0.74 & \\
\hline \multicolumn{6}{|l|}{ Adjustment for confounding factors ${ }^{3}$} \\
\hline \multirow[t]{2}{*}{ Age } & 8 & $0.75(0.69-0.81)$ & 0 & 0.94 & $\mathrm{NC}$ \\
\hline & 0 & & & & \\
\hline
\end{tabular}




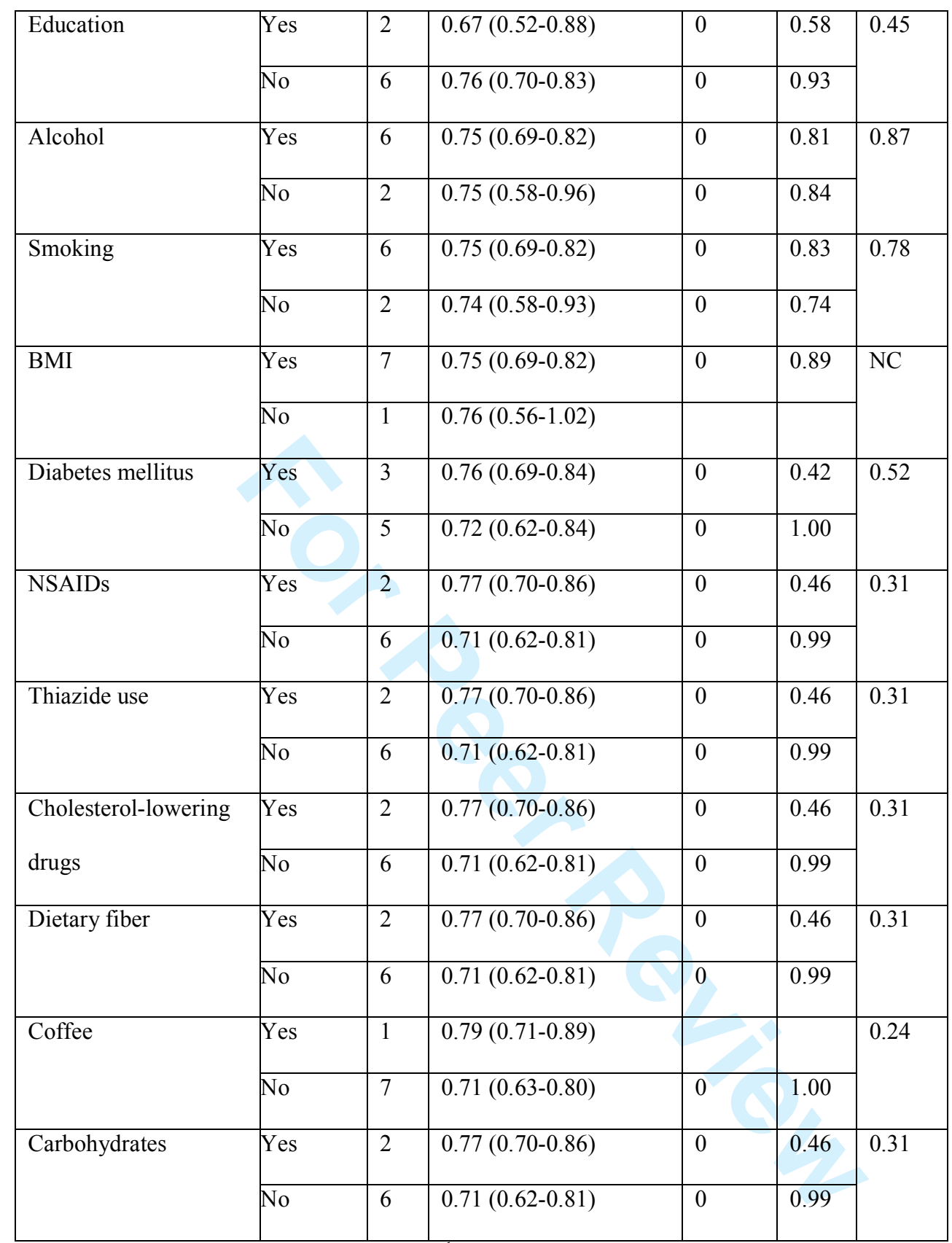

$n$ denotes the number of studies, ${ }^{1} \mathrm{P}$ for heterogeneity within each subgroup,

${ }^{2} \mathrm{P}$ for heterogeneity between subgroups with meta-regression analysis

$\mathrm{NC}$, not calculable because no studies were present in one of the subgroups. 
Figure 1. Flow-chart of study selection

\section{7 records identified in total: 7799 records identified in PubMed 4948 records identified in Embase}

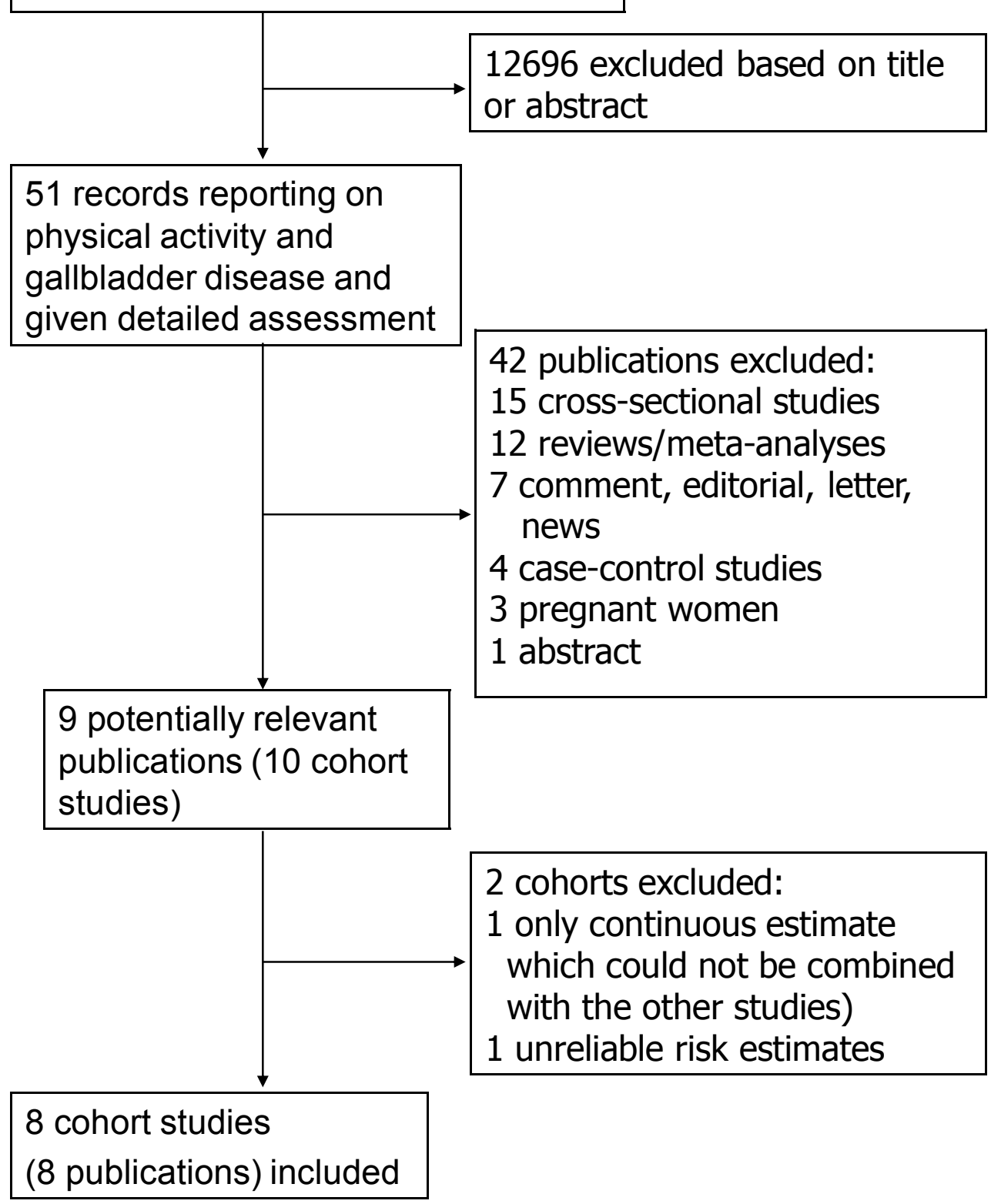


Figure 2. Physical activity and gallbladder disease, highest versus lowest comparison

Study

Banim, 2010

Williams, 2008

Storti, 2005

Boland, 2002

Leitzmann, 1999

Leitzmann, 1998

Sahi, 1998

Kato, 1992

Overall

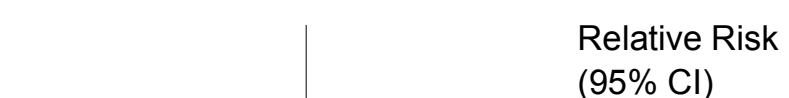

$(95 \% \mathrm{Cl})$

$0.70(0.48,1.01)$

$0.73(0.49,1.10)$

$0.63(0.44,0.90)$

$0.76(0.56,1.02)$

$0.79(0.71,0.89)$

$0.72(0.58,0.90)$

$0.72(0.46,1.13)$

$0.70(0.60,1.00)$

$0.75(0.69,0.81)$

\section{5}

.25


Supplementary text.

Embase search terms

1) (body mass index OR BMI OR overweight OR obesity OR anthropometry OR fatness OR body fatness OR abdominal fatness OR abdominal obesity OR waist circumference OR waistto-hip ratioORphysical activity OR exercise OR sports OR walking OR biking OR running OR fitness OR exercise test OR inactivityOR diabetesOR smoking OR tobaccoOR risk factor OR risk factors).ti,ab.

2) body mass index/ OR BMI/ OR overweight/ OR obesity/ OR anthropometry/ OR fatness/ OR body fatness/ OR abdominal fatness/ OR abdominal obesity/ OR waist circumference/ OR waist-to-hip ratio/ ORphysical activity/ OR exercise/ OR sports/ OR walking/ OR biking/ OR running/ OR fitness/ OR exercise test/ OR inactivity/ OR diabetes/ OR smoking/ OR tobacco/ OR risk factor/ OR risk factors/

3) (gallstones OR gallbladder disease OR cholelithiasis OR cholecystectomy ORcholecystitis).ti,ab.

4) gallstones/ OR gallbladder disease/ OR cholelithiasis/ OR cholecystectomy/ OR cholecystitis/

5) (case-control or retrospective or cohort or cohorts or prospective or longitudinal or followup or cross-sectional or trial or relative risk or risk or hazard ratio or odds ratio or regression).ti.ab.

6) 1 OR 2

7) 3 OR 4

8) 5 AND 6 AND 7

9) limit 8 to human 
Supplementary Table 1

\begin{tabular}{|l|l|}
\hline Exclusion reason & Reference number \\
\hline Abstract & $(1)$ \\
\hline Case-control study & $(2-5)$ \\
\hline Cross-sectional study & $(6-20)$ \\
\hline Gallstones in pregnancy & $(21-23)$ \\
\hline Letter/editorial/news/note/comment & $(24-30)$ \\
\hline Meta-analysis & $(31)$ \\
\hline $\begin{array}{l}\text { Review } \\
\text { Not usable result, only continuous } \\
\text { estimate (square root of the physical } \\
\text { activity level was used). We were not } \\
\text { able to obtain supplementary } \\
\text { (categorical) data from the authors. }\end{array}$ & $(43)$ \\
\hline
\end{tabular}

Reference List

1. Pavey T, Kolbe-Alexander T, Peeters G, Brown W. Physical activity and surgery in mid-aged women. Journal of Science and Medicine in Sport 2013;Conference:December.

2. Misciagna $\mathrm{G}$, Centonze $\mathrm{S}$, Leoci $\mathrm{C}$ et al. Diet, physical activity, and gallstones--a populationbased, case-control study in southern Italy. Am J Clin Nutr 1999;69:120-6.

3. Sachdeva S, Khan Z, Ansari MA, Khalique N, Anees A. Lifestyle and gallstone disease: scope for primary prevention. Indian J Community Med 2011;36:263-7.

4. Paracha PI, Asif Y, Vriesekoop F et al. Risk factors associated with gallstone disease in women. e-SPEN Journal 2012;7:June.

5. Niiyama H, Mizumoto K, Sato $\mathrm{N}$ et al. Physical activity, biliary lipids, and gallstones in obese subjects. American Journal of Gastroenterology 2001;96:2001.

6. Henao-Moran S, ova-Gutierrez E, Moran S et al. Recreational physical activity is inversely associated with asymptomatic gallstones in adult Mexican women. Annals of Hepatology 2014;13:01.

7. Williams CN, Johnston JL. Prevalence of gallstones and risk factors in Caucasian women in a rural Canadian community. Can Med Assoc J 1980;122:664-8. 
8. Jorgensen T. Gall stones in a Danish population. Relation to weight, physical activity, smoking, coffee consumption, and diabetes mellitus. Gut 1989;30:528-34.

9. Kono S, Shinchi K, Todoroki I et al. Gallstone disease among Japanese men in relation to obesity, glucose intolerance, exercise, alcohol use, and smoking. Scand J Gastroenterol 1995;30:372-6.

10. Everhart JE, Khare M, Hill M, Maurer KR. Prevalence and ethnic differences in gallbladder disease in the United States. Gastroenterology 1999;117:632-9.

11. Chuang CZ, Martin LF, LeGardeur BY, Lopez A. Physical activity, biliary lipids, and gallstones in obese subjects. Am J Gastroenterol 2001;96:1860-5.

12. Everhart JE, Yeh F, Lee ET et al. Prevalence of gallbladder disease in American Indian populations: findings from the Strong Heart Study. Hepatology 2002;35:1507-12.

13. Volzke H, Baumeister SE, Alte D et al. Independent risk factors for gallstone formation in a region with high cholelithiasis prevalence. Digestion 2005;71:97-105.

14. Sakuta H, Suzuki T. Plasma total homocysteine and gallstone in middle-aged Japanese men. J Gastroenterol 2005;40:1061-4.

15. Escobar V, Oakes SL, Wood R, Becho J, Markides K, Espino DV. Prevalence and characteristics associated with self-reported gall bladder disease in Mexican American elders: results from the Hispanic Established Populations for Epidemiologic Studies in the Elderly (HEPESE). Aging Clin Exp Res 2009;21:33-7.

16. Walcher $\mathrm{T}$, Haenle MM, Kron $\mathrm{M}$ et al. Vitamin $\mathrm{C}$ supplement use may protect against gallstones: an observational study on a randomly selected population. BMC Gastroenterol 2009;9:74.

17. Hou L, Shu XO, Gao YT et al. Anthropometric measurements, physical activity, and the risk of symptomatic gallstone disease in Chinese women. Ann Epidemiol 2009;19:344-50.

18. Lee Y-C, Wu J-S, Yang Y-C, Cang C-S, Lu F-H, Chang C-J. Hepatitis B and hepatitis C associated with risk of gallstone disease in elderly adults. Journal of the American Geriatrics Society 2014;62:August.

19. Chen YC, Chiou C, Lin MN, Lin CL. The prevalence and risk factors for gallstone disease in taiwanese vegetarians. PLoS One 2014;9:e115145.

20. Lee Y-C, Wu J-S, Yang Y-C, Chang C-S, Lu F-H, Chang C-J. Moderate to severe, but not mild, nonalcoholic fatty liver disease associated with increased risk of gallstone disease. Scandinavian Journal of Gastroenterology 2014;49:August.

21. Ko CW, Napolitano PG, Lee SP, Schulte SD, Ciol MA, Beresford SA. Physical Activity, Maternal Metabolic Measures, and the Incidence of Gallbladder Sludge or Stones during Pregnancy: A Randomized Trial. Am J Perinatol 2013.

22. Lindseth G, Bird-Baker MY. Risk factors for cholelithiasis in pregnancy. Res Nurs Health 2004;27:382-91.

23. Basso L, McCollum PT, Darling MR, Tocchi A, Tanner WA. A descriptive study of pregnant women with gallstones. Relation to dietary and social habits, education, physical activity, height, and weight. Eur J Epidemiol 1992;8:629-33. 
24. Vega KJ, Johnston DE. Exercise and the gallbladder. N Engl J Med 1999;341:836-7.

25. Hofmann AF. Recreational physical activity and the risk of cholecystectomy in women. N Engl J Med 2000;342:213-4.

26. Lawlor DA, Hanratty B. Recreational physical activity and the risk of cholecystectomy in women. N Engl J Med 2000;342:212-4.

27. Simini B. Recreational physical activity and the risk of cholecystectomy in women. N Engl J Med 2000;342:212-3.

28. Roseau E. [Is physical activity a factor in reducing risk of gallbladder calculi in the woman?]. Presse Med 1999;28:2042-3.

29. Roseau E. Does physical activity reduce the risk of gallbladder lithiasis?. [French]. Presse Medicale 1999;28:27.

30. Leitzmann MF, Rimm EB, Willett WC. Physical activity reduces risk for cholecystectomy in women. Consultant 2000;40:2000.

31. Shi J, Liu S, Xie W. Risk factors of cholelithiasis in China: A meta-analysis. [Chinese]. Chinese Journal of Gastroenterology 2005;10:2005.

32. Colditz GA. Economic costs of obesity and inactivity. Medicine and Science in Sports and Exercise 1999;31:1999.

33. Rissanen A, Fogelholm M. Physical activity in the prevention and treatment of other morbid conditions and impairments associated with obesity: current evidence and research issues. Med Sci Sports Exerc 1999;31:S635-S645.

34. Peters HPF, De Vries WR, VanBerge-Henegouwen GP, Akkermans LMA. Potential benefits and hazards of physical activity and exercise on the gastrointestinal tract. Gut 2001;48:2001.

35. Trevisani F, Scarpa M, Di MA. Role of physical activity in the prevention of gastrointestinal diseases. [Italian]. Medicina dello Sport 2005;58:September.

36. Shaffer EA. Gallstone disease: Epidemiology of gallbladder stone disease. Best Pract Res Clin Gastroenterol 2006;20:981-96.

37. Shaffer EA. Epidemiology of gallbladder stone disease. Best Practice and Research: Clinical Gastroenterology 2006;20:2006.

38. de Oliveira EP, Burini RC. The impact of physical exercise on the gastrointestinal tract. Curr Opin Clin Nutr Metab Care 2009;12:533-8.

39. Stinton LM, Myers RP, Shaffer EA. Epidemiology of gallstones. Gastroenterol Clin North Am 2010;39:157-69, vii.

40. Martin D. Physical activity benefits and risks on the gastrointestinal system. Southern Medical Journal 2011;104:December.

41. Stinton LM, Shaffer EA. Epidemiology of gallbladder disease: cholelithiasis and cancer. Gut Liver 2012;6:172-87.

42. Booth FW, Roberts CK, Laye MJ. Lack of exercise is a major cause of chronic diseases. Compr Physiol 2012;2:1143-211. 
43. Kriska AM, Brach JS, Jarvis BJ et al. Physical activity and gallbladder disease determined by ultrasonography. Med Sci Sports Exerc 2007;39:1927-32. 
Supplementary Table 2. Leisure-time physical activity and gallstones, nonlinear dose-response

\begin{tabular}{|c|c|c|c|c|c|c|}
\hline & \multicolumn{2}{|c|}{ Leisure-time physical activity } & \multicolumn{2}{|c|}{ Vigorous physical activity } & \multicolumn{2}{|c|}{ Non-vigorous physical activity } \\
\hline & Not BMI adjusted & BMI adjusted & Not BMI adjusted & BMI adjusted & Not BMI adjusted & BMI adjusted \\
\hline MET-hrs per week & $\mathrm{RR}(95 \% \mathrm{CI})$ & $\mathrm{RR}(95 \% \mathrm{CI})$ & $\mathrm{RR}(95 \% \mathrm{CI})$ & $\mathrm{RR}(95 \% \mathrm{CI})$ & $\mathrm{RR}(95 \% \mathrm{CI})$ & $\mathrm{RR}(95 \% \mathrm{CI})$ \\
\hline 1 & 1.00 & 1.00 & 1.00 & 1.00 & 1.00 & 1.00 \\
\hline 5 & $0.89(0.83-0.96)$ & $0.93(0.87-1.00)$ & $0.92(0.84-1.00)$ & $0.95(0.89-1.03)$ & $0.91(0.85-0.99)$ & $0.92(0.88-0.97)$ \\
\hline 10 & $0.83(0.76-0.91)$ & $0.89(0.81-0.97)$ & $0.89(0.81-0.97)$ & $0.93(0.87-1.00)$ & $0.86(0.79-0.93)$ & $0.84(0.76-0.94)$ \\
\hline 15 & $0.78(0.72-0.86)$ & $0.85(0.78-0.93)$ & $0.84(0.77-0.91)$ & $0.89(0.84-0.95)$ & $0.84(0.77-0.91)$ & $0.97(0.87-1.08)$ \\
\hline 20 & $0.75(0.68-0.81)$ & $0.82(0.76-0.90)$ & $0.78(0.71-0.86)$ & $0.84(0.78-0.91)$ & & \\
\hline 25 & $0.71(0.65-0.78)$ & $0.80(0.73-0.87)$ & $0.71(0.63-0.80)$ & $0.78(0.70-0.87)$ & & \\
\hline 30 & $0.68(0.62-0.74)$ & $0.77(0.71-0.84)$ & $0.63(0.54-0.75)$ & $0.71(0.61-0.84)$ & & \\
\hline 35 & $0.65(0.59-0.72)$ & $0.75(0.68-0.83)$ & & & & \\
\hline 40 & $0.62(0.55-0.70)$ & $0.73(0.65-0.81)$ & & & & \\
\hline
\end{tabular}


Supplementary Figure 1. Funnel plot

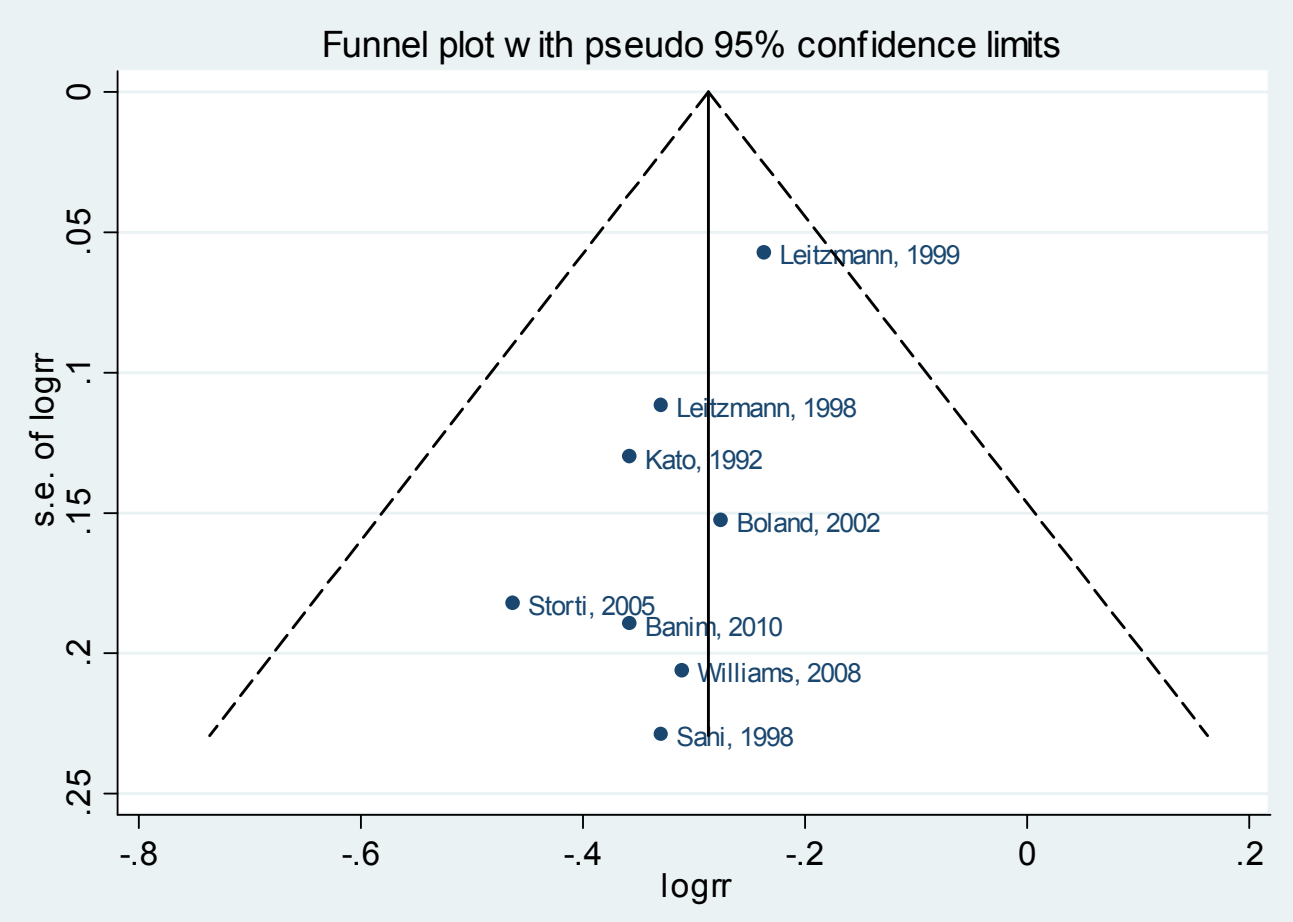

Human Kinetics, 1607 N Market St, Champaign, IL 61825 
Supplementary Figure 2. Leisure-time physical activity and gallbladder disease, with and without BMI adjustment

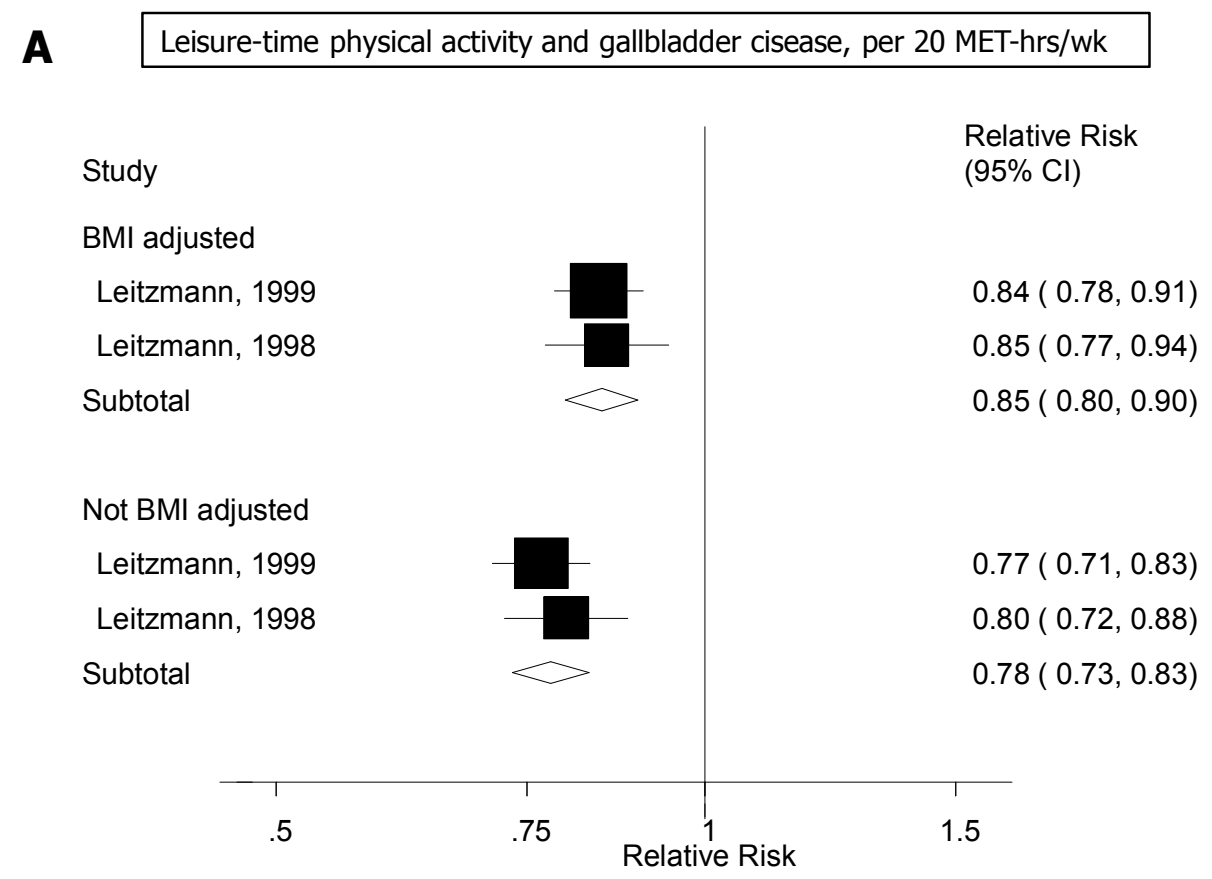

B Leisure-time physical activity and gallbladder cisease, non-linear dose-response

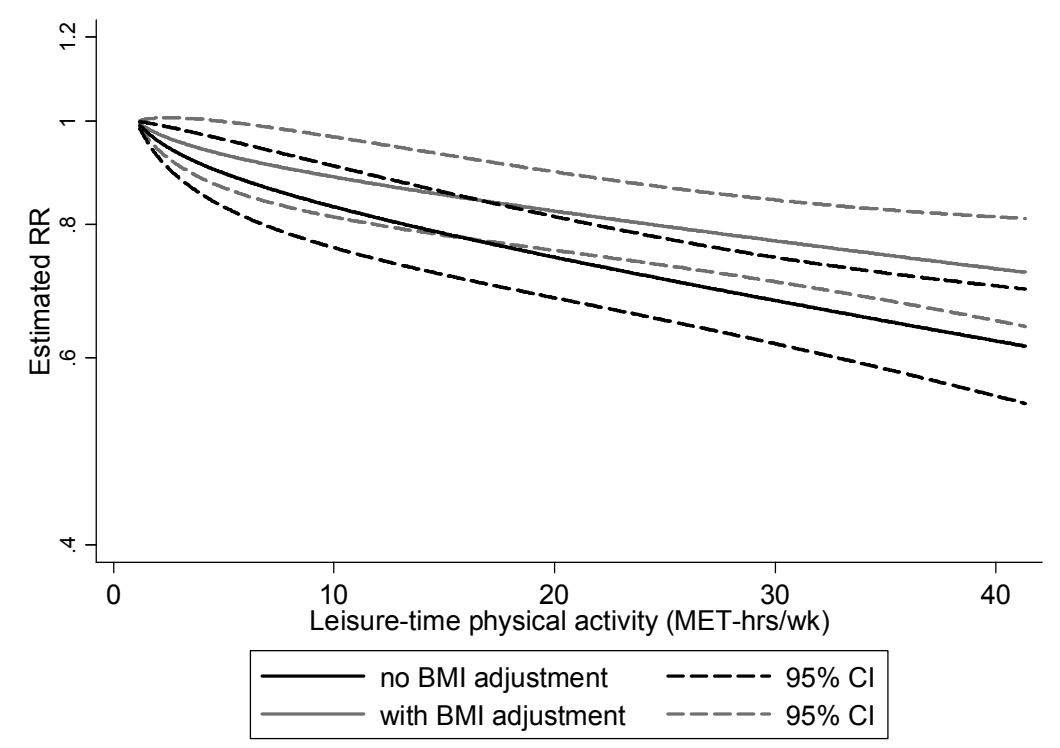

Human Kinetics, 1607 N Market St, Champaign, IL 61825 
1

2

3

4

5

6

7

8

9

10

11

12

13

14

15

16

17

18

19

20

21

22

23

24

25

26

27

28

29

30

31

32

33

34

35

36

37

38

39

40

41

42

43

44

45

46

47

48

49

50

51

52

53

54

55

56

57

58

59

60

Supplementary Figure 3. Leisure-time physical activity and gallbladder disease, per 1000 kcal/week

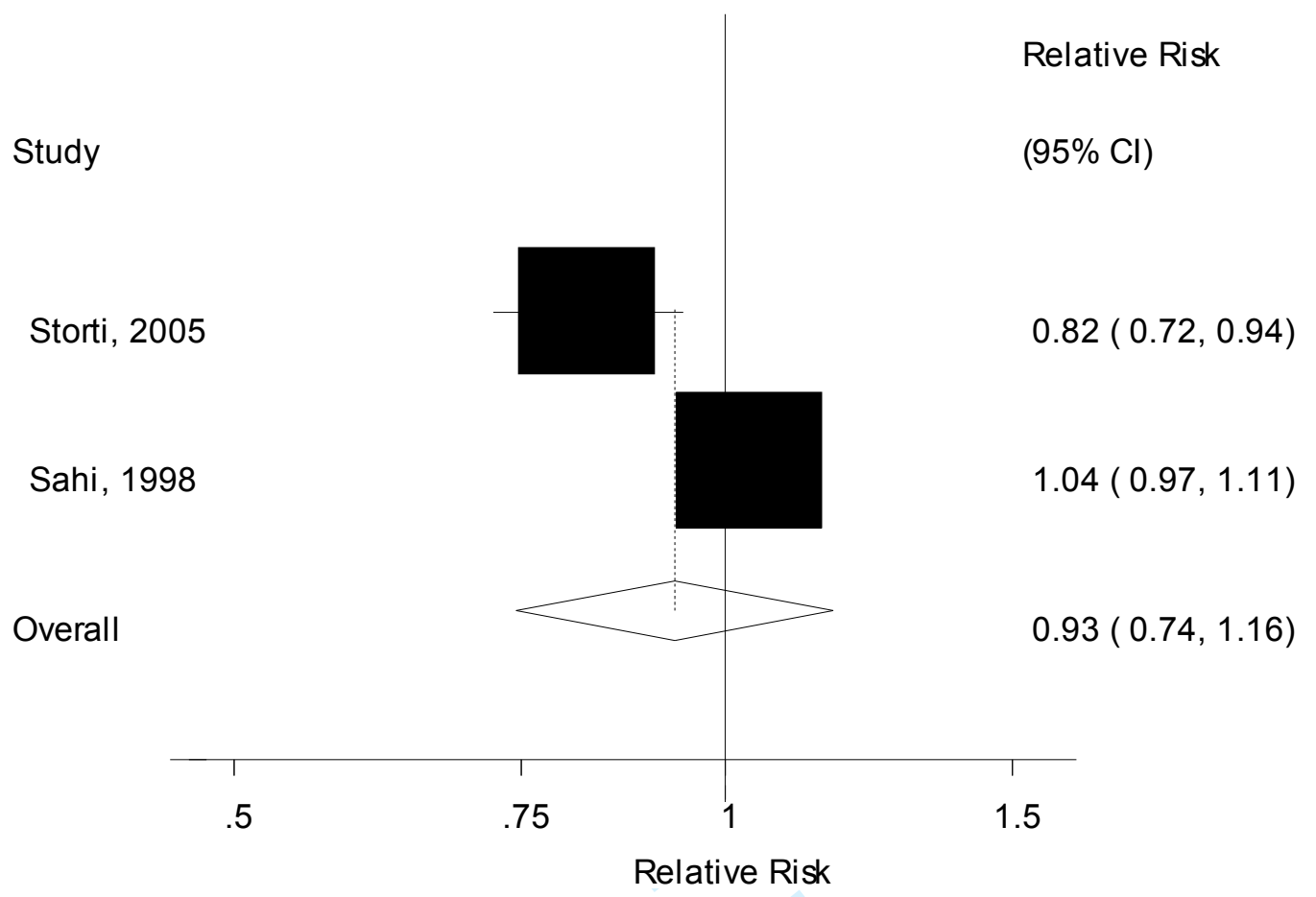

Human Kinetics, 1607 N Market St, Champaign, IL 61825 
Supplementary Figure 4. Vigorous physical activity and gallbladder disease, with and without BMI adjustment

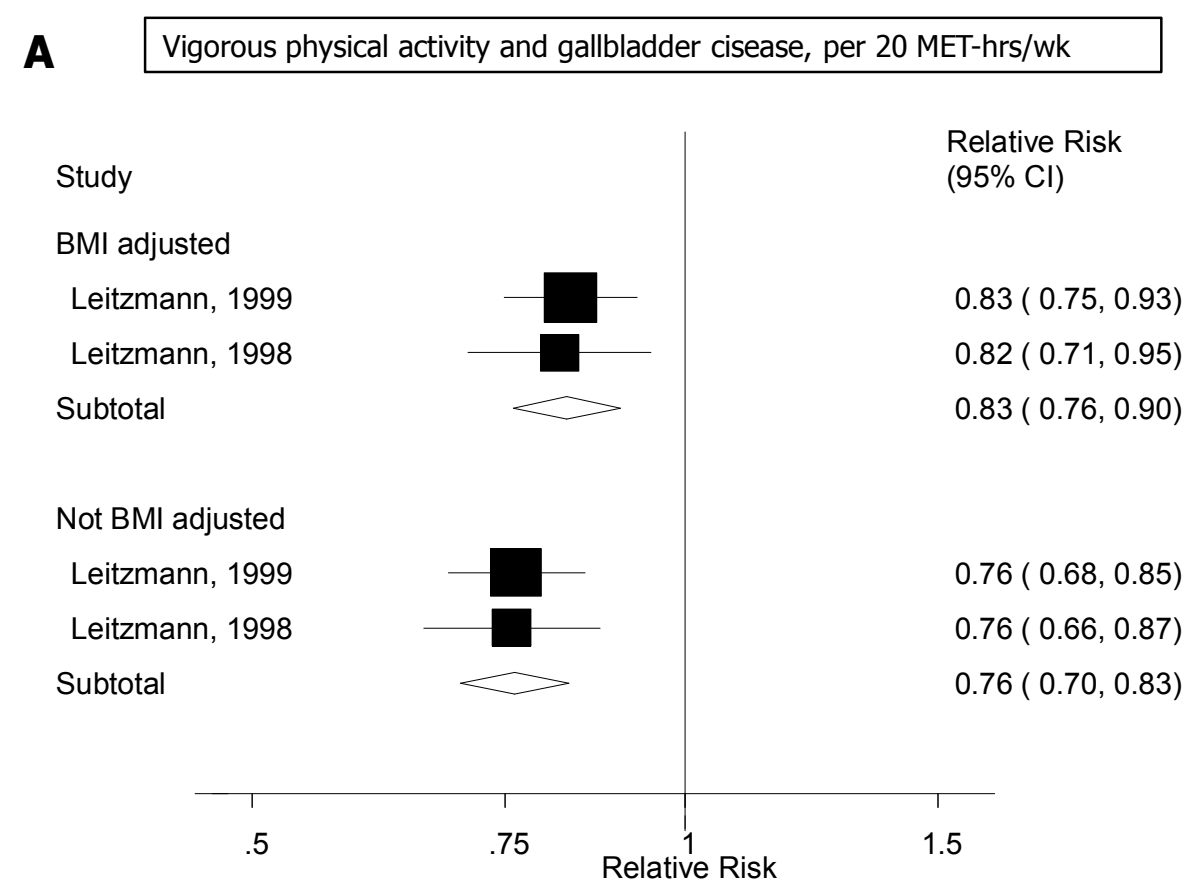

B Vigorous physical activity and gallbladder cisease, non-linear dose-response

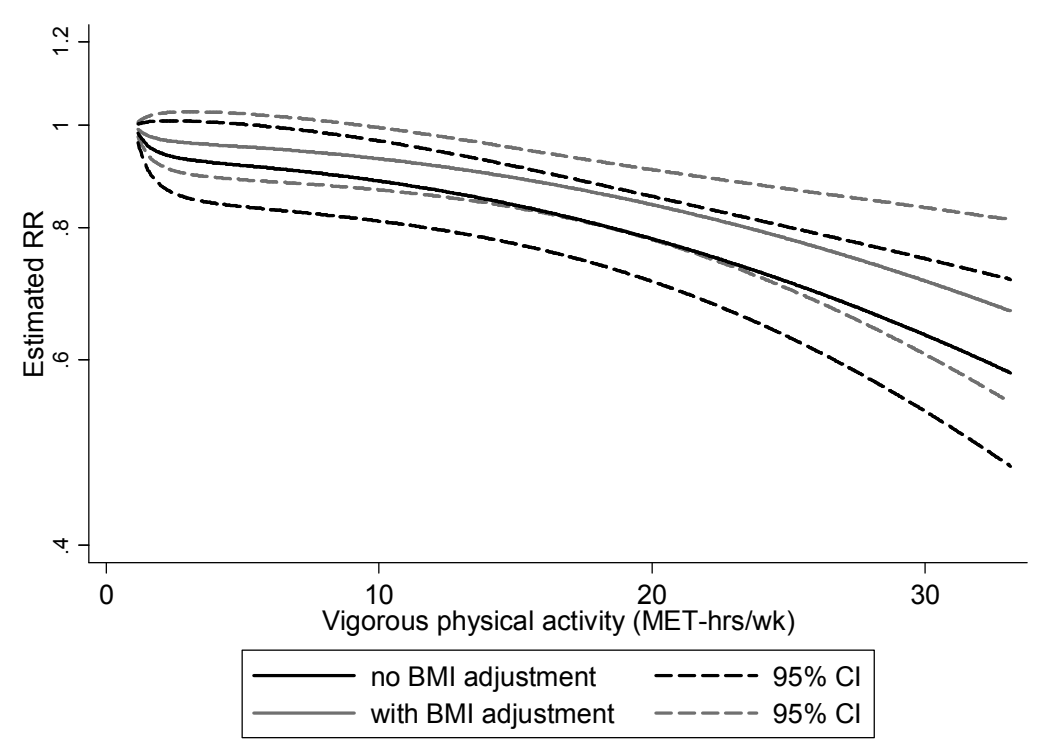

Human Kinetics, 1607 N Market St, Champaign, IL 61825 
Supplementary Figure 5. Nonvigorous physical activity and gallbladder disease, with and without BMI adjustment

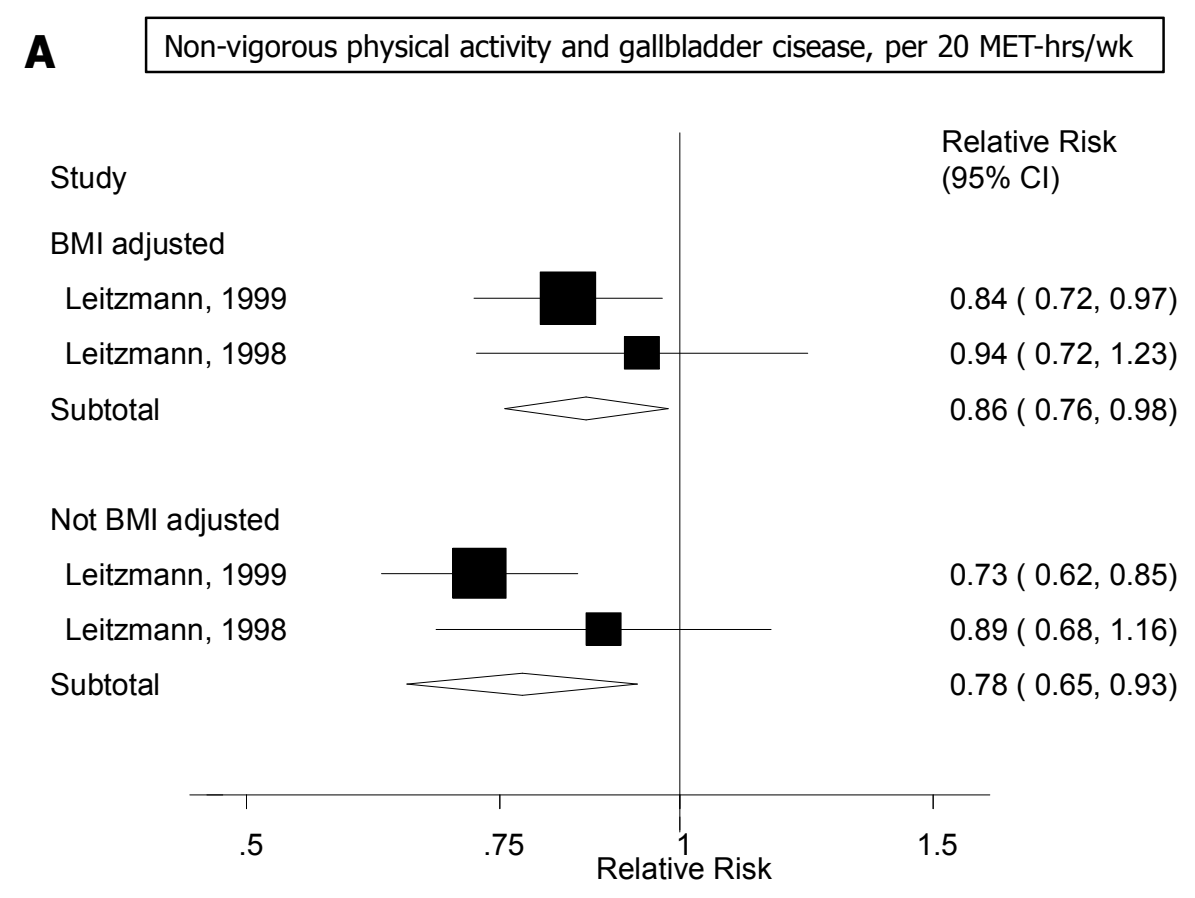

B Non-vigorous physical activity and gallbladder cisease, non-linear dose-response

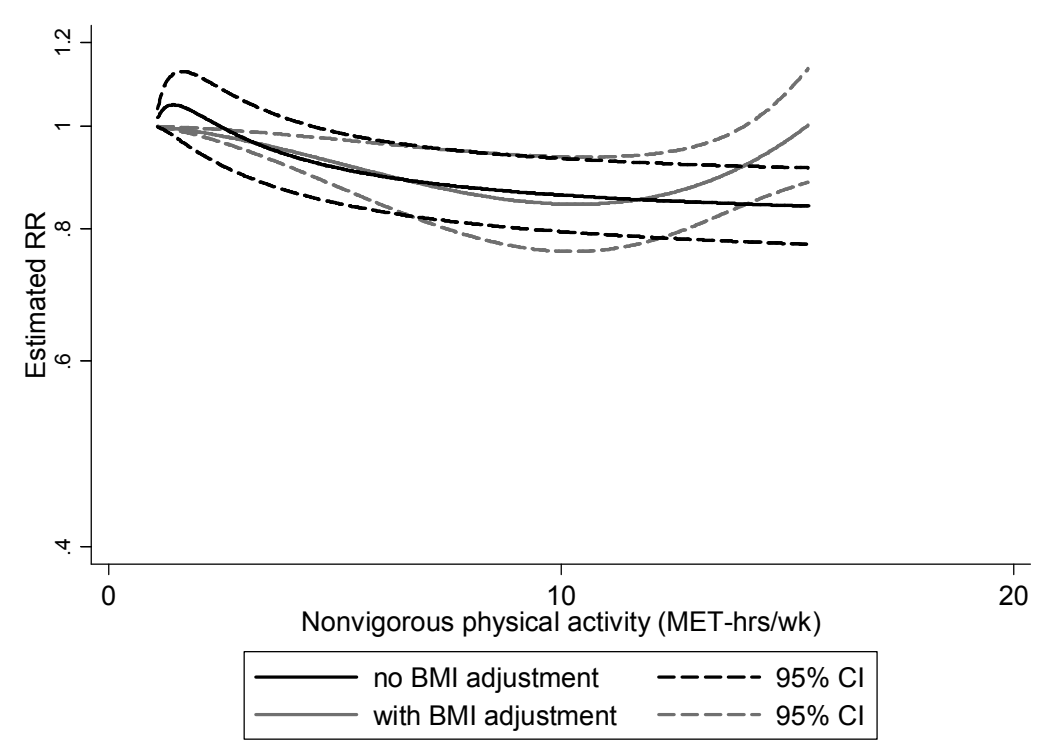




\section{ABSTRACT}

12 Structured summary $1 \beta$

14

1

\section{INTRODUCTION}

\section{Rationale}

$1 \$$ Objectives

20

\section{1}

\section{METHODS}

23 Protocol and registration

24

25 Eligibility criteria

27

28 Information sources

29

30

Search

$$
32
$$

33

34

35

36

3

38

39

40

41

42 Risk of bias in individual

43 studies

44 Summary measures
\# Checklist item

1 Identify the report as a systematic review, meta-analysis, or both.

2 Provide a structured summary including, as applicable: background; objectives; data sources; study eligibility criteria, participants, and interventions; study appraisal and synthesis methods; results; limitations; conclusions and implications of key findings; systematic review registration number.

3 Describe the rationale for the review in the context of what is already known

3

4 Provide an explicit statement of questions being addressed with reference to participants, interventions, comparisons, 3 outcomes, and study design (PICOS).

5 Indicate if a review protocol exists, if and where it can be accessed (e.g., Web address), and, if available, provide registration information including registration number.

6 Specify study characteristics (e.g., PICOS, length of follow-up) and report characteristics (e.g., years considered, language, publication status) used as criteria for eligibility, giving rationale.

7 Describe all information sources (e.g., databases with dates of coverage, contact with study authors to identify additional studies) in the search and date last searched.

8 Present full electronic search strategy for at least one database, including any limits used, such that it could be repeated.

9 State the process for selecting studies (i.e., screening, eligibility, included in systematic review, and, if applicable, included in the meta-analysis).

10 Describe method of data extraction from reports (e.g., piloted forms, independently, in duplicate) and any processes for obtaining and confirming data from investigators.

11 List and define all variables for which data were sought (e.g., PICOS, funding sources) and any assumptions and simplifications made.

12 Describe methods used for assessing risk of bias of individual studies (including specification of whether this was done at the study or outcome level), and how this information is to be used in any data synthesis.

13 State the principal summary measures (e.g., risk ratio, difference in means).

Human Kinetics, 1607 N Market St, Champaign, IL 61825

\section{No} protocol

4,5

4

4

4,5 ,

flowchart

- figure 1

5

5

7

5,6 


\section{RESULTS}

10

14 Describe the methods of handling data and combining results of studies, if done, including measures of consistency (e.g., $I^{2}$ ) for each meta-analysis.

\section{Page 1 of 2}

\section{\# Checklist item}

Reported on

15 Specify any assessment of risk of bias that may affect the cumulative evidence (e.g., publication bias, selective 7 reporting within studies).

16 Describe methods of additional analyses (e.g., sensitivity or subgroup analyses, meta-regression), if done, indicating which were pre-specified.

tudy selection

17 Give numbers of studies screened, assessed for eligibility, and included in the review, with reasons for exclusions at each stage, ideally with a flow diagram.

18 For each study, present characteristics for which data were extracted (e.g., study size, PICOS, follow-up period) and provide the citations.

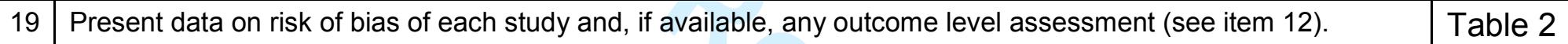

20 For all outcomes considered (benefits or harms), present, for each study: (a) simple summary data for each intervention group (b) effect estimates and confidence intervals, ideally with a forest plot.

21 Present results of each meta-analysis done, including confidence intervals and measures of consistency.

22 Present results of any assessment of risk of bias across studies (see Item 15).

23 Give results of additional analyses, if done (e.g., sensitivity or subgroup analyses, meta-regression [see Item 16]).

Table 1
Table 1

Table 1

Table 1

Additional analysis

\section{Figure 2,} Supplementary Figure 2-5 8-10, Supplementary figure 1 9-10, Table 2

24 Summarize the main findings including the strength of evidence for each main outcome; consider their relevance to key groups (e.g., healthcare providers, users, and policy makers).

25 Discuss limitations at study and outcome level (e.g., risk of bias), and at review-level (e.g., incomplete retrieval of identified research, reporting bias).

26 Provide a general interpretation of the results in the context of other evidence, and implications for future research. Human Kinetics, 1607 N Market St, Champaign, IL 61825 


\begin{tabular}{l|l|l|l|l|}
\hline & \multicolumn{2}{|l|}{ FUNDING } \\
5 \\
6 & Funding & 27 & $\begin{array}{l}\text { Describe sources of funding for the systematic review and other support (e.g., supply of data); role of funders } \\
\text { for the systematic review. }\end{array}$ & 14 \\
\hline
\end{tabular}

9 From: Moher D, Liberati A, Tetzlaff J, Altman DG, The PRISMA Group (2009). Preferred Reporting Items for Systematic Reviews and Meta-Analyses: The PRISMA Statement. PLoS Med 6(6): e1000097. 10 doi:10.1371/journal.pmed1000097 